\title{
Mandatory Versus Discretionary Spending: the Status Quo Effect
}

\author{
Online Appendix
}

T. Renee Bowen*

Ying Chen $^{\dagger}$

Hülya Eraslan ${ }^{\ddagger}$

January 24, 2014

* Stanford University and the Hoover Institution

$\dagger$ Johns Hopkins University and University of Southampton

$\ddagger$ Johns Hopkins University 


\section{B1. Pareto efficient allocations}

PROPOSITION B.1: Consider a T-period problem where $T \in\{0,1, \ldots, \infty\}$. For any Pareto efficient allocation in the T-period problem such that $x_{L}^{t^{\prime}}>0$ for some $t^{\prime} \leq T$ and $x_{H}^{t^{\prime \prime}}$ for some $t^{\prime \prime} \leq T$, we have $g^{t}=\theta_{H}+\theta_{L}$ for all $t \leq T$.

Proof: A Pareto efficient allocation in the $T$-period problem solves the following problem:

$$
\begin{aligned}
\max _{\left\{\mathbf{b}^{t}\right\}_{t=0}^{\infty}} & \sum_{t=0}^{T} \delta^{t}\left(x_{L}^{t}+\theta_{L} \ln \left(g^{t}\right)\right) \\
\text { s.t. } & \sum_{t=0}^{T} \delta^{t}\left(x_{H}^{t}+\theta_{H} \ln \left(g^{t}\right)\right)=\bar{U}, \\
& x_{L}^{t}+x_{H}^{t}+g^{t} \leq 1, x_{L}^{t} \geq 0, x_{H}^{t} \geq 0, g^{t} \geq 0 \text { for all } t \leq T .
\end{aligned}
$$

We first prove the result for any finite $T$. The Lagrangian of this problem is

$$
\sum_{t=0}^{T} \delta^{t}\left(x_{L}^{t}+\theta_{L} \ln \left(g^{t}\right)\right)-\lambda_{1}\left(\bar{U}-\sum_{t=0}^{T} \delta^{t}\left(x_{H}^{t}+\theta_{H} \ln \left(g^{t}\right)\right)\right)-\sum_{t=0}^{T} \lambda_{2 t}\left(x_{L}^{t}+x_{H}^{t}+g^{t}-1\right) .
$$

The first order conditions with respect to $x_{L}^{t}, x_{H}^{t}$ and $g^{t}$ are:

$$
\begin{aligned}
\delta^{t}-\lambda_{2 t} & \leq 0, & & \left(\delta^{t}-\lambda_{2 t}\right) x_{L}^{t}=0, \\
\lambda_{1} \delta^{t}-\lambda_{2 t} & \leq 0, & & \left(\lambda_{1} \delta^{t}-\lambda_{2 t}\right) x_{H}^{t}=0, \\
\frac{\delta^{t} \theta_{L}}{g^{t}}+\frac{\lambda_{1} \delta^{t} \theta_{H}}{g^{t}}-\lambda_{2 t} & \leq 0, & & \left(\frac{\delta^{t} \theta_{L}}{g^{t}}+\frac{\lambda_{1} \delta^{t} \theta_{H}}{g^{t}}-\lambda_{2 t}\right) g^{t}=0 .
\end{aligned}
$$

Suppose there exist $t^{\prime}$ and $t^{\prime \prime}$ such $x_{L}^{t^{\prime}}>0$ and $x_{H}^{t^{\prime \prime}}>0$. Since $x_{L}^{t^{\prime}}>0$, we have $\lambda_{2 t^{\prime}}=\delta^{t^{\prime}}$ from (B1). It follows from (B2) that $\lambda_{1} \leq \frac{\lambda_{2 t^{\prime}}}{\delta^{t^{\prime}}}=1$. Similarly, since $x_{H}^{t^{\prime \prime}}>0$, we have $\lambda_{1}=\frac{\lambda_{2 t^{\prime \prime}}}{\delta^{t^{\prime \prime}}}$. Since $\frac{\lambda_{2 t^{\prime \prime}}}{\delta t^{\prime \prime}} \geq 1$ from (B1), it follows that $\lambda_{1} \geq 1$. Hence $\lambda_{1}=1$.

Note that $g^{t}=0$ violates (B3), hence $g^{t}>0$. We next show that $g^{t} \neq 1$ for any $t \leq T$, which implies that for any $t \leq T$, at least one of $x_{H}^{t}$ and $x_{L}^{t}$ is strictly positive. Suppose $g^{t}=1$ for some $t$, then, since $\lambda_{1}=1$, (B3) implies that $\lambda_{2 t}=\delta^{t}\left(\theta_{H}+\lambda_{1} \theta_{L}\right)<\delta^{t}$, which contradicts (B1). Since $\lambda_{1}=1$ and at least one of $x_{L}^{t}$ and $x_{H}^{t}$ is strictly positive for any $t \leq T$, it follows that $\lambda_{2 t}=\delta^{t}$ for all $t \leq T$. Substituting in (B3), we get $g^{t}=\theta_{H}+\theta_{L}$ for all $t \leq T$.

Now suppose $T=\infty$. Consider an allocation $\mathbf{A}=\left\{\mathbf{b}^{t}\right\}_{t=0}^{\infty}$ in which $x_{L}^{t^{\prime}}>0$ for some $t^{\prime}, x_{H}^{t^{\prime \prime}}$ for some $t^{\prime \prime}$ and $g^{\tilde{t}} \neq \theta_{H}+\theta_{L}$ for some $\tilde{t}$. We next show that this allocation is not Pareto efficient. Let $\tau=\max \left\{t^{\prime}, t^{\prime \prime}, \tilde{t}\right\}$. Then, as we have shown above, $\left\{\mathbf{b}^{t}\right\}_{t=0}^{\tau}$ is not a Pareto efficient allocation in the $\tau$-period problem. Let $\left\{\mathbf{b}_{t}^{\prime}\right\}_{t=0}^{\tau}$ be an allocation that Pareto dominates $\left\{\mathbf{b}^{t}\right\}_{t=0}^{\tau}$ in the $\tau$-period problem. Let $\mathbf{A}^{\prime \prime}=\left\{\mathbf{b}_{t}^{\prime \prime}\right\}_{t=0}^{\infty}$ be such that $\mathbf{b}_{t}^{\prime \prime}=\mathbf{b}_{t}^{\prime}$ for $t \leq \tau$ and $\mathbf{b}_{t}^{\prime \prime}=\mathbf{b}^{t}$ for $t>\tau$. It follows that $\mathbf{A}^{\prime \prime}$ Pareto dominates $\mathbf{A}$. Hence $\mathbf{A}$ is not Pareto efficient, which establishes the result for $T=\infty$. 


\section{B2. Non-Markov equilibrium with discretionary public spending}

Consider the following strategy profile: (i) In period 0 , if $i$ is the proposer, then it proposes $\mathbf{z}=\left(g^{\prime}, x_{H}^{\prime}, x_{L}^{\prime}\right)$ with $g^{\prime}=\theta_{H}+\theta_{L}, x_{i}^{\prime}=1-g^{\prime}$ and $x_{j}^{\prime}=0$; (ii) if the level of public good spending proposed in all previous periods is equal to $\theta_{H}+\theta_{L}$, then proposer $i$ in period $t$ proposes $g^{\prime}=\theta_{H}+\theta_{L}, x_{i}^{\prime}=1-g^{\prime}, x_{j}^{\prime}=0$; (iii) if the level of public good spending proposed in some previous period is not equal to $\theta_{H}+\theta_{L}$, then proposer $i$ in period $t$ proposes $g^{\prime}=\theta_{i}, x_{i}^{\prime}=1-g^{\prime}, x_{j}^{\prime}=0$; (iv) party $j$ accepts a proposal if and only if its dynamic payoff from accepting is weakly higher than its dynamic payoff from rejecting.

Off the equilibrium path, proposer $i$ has no incentive to deviate because the strategies induce a Markov perfect equilibrium in subgames off the equilibrium path by Proposition 1.

On the equilibrium path, proposer $i$ 's payoff from following the strategy is given by

$$
V_{i}^{*}=\frac{(1-\delta p)\left(1-\left(\theta_{H}+\theta_{L}\right)\right)+\theta_{i} \ln \left(\theta_{H}+\theta_{L}\right)(1+\delta-2 \delta p)}{(1-\delta)(1+\delta-2 \delta p)} .
$$

If $i$ deviates, then the most profitable deviation is to propose $g^{\prime}=\theta_{i}, x_{i}^{\prime}=1-g^{\prime}, x_{j}^{\prime}=0$ and the payoff from this deviation is given by

$$
V_{i}=\frac{(1-\delta p)\left(1-\theta_{i}\right)+\theta_{i} \ln \left(\theta_{i}\right)(1-\delta p)+\theta_{i} \ln \left(\theta_{j}\right) \delta(1-p)}{(1-\delta)(1+\delta-2 \delta p)} .
$$

Proposer has $i$ has no incentive to deviate if $V_{i}^{*} \geq V_{i}$. This is satisfied when

$$
\ln \left(\frac{\theta_{H}+\theta_{L}}{\theta_{i}}\right)+\frac{\delta(1-p)}{1-\delta p} \ln \left(\frac{\theta_{H}+\theta_{L}}{\theta_{j}}\right) \geq \frac{\theta_{j}}{\theta_{i}}
$$

which in turn is satisfied when $\delta \geq \underline{\delta}_{i}$ where $\underline{\delta}_{i}=\frac{Z_{i}}{Z_{i} p+1-p}$ and $Z_{i}=\frac{\frac{\theta_{j}}{\theta_{i}}-\ln \left(\frac{\theta_{H}+\theta_{L}}{\theta_{i}}\right)}{\ln \left(\frac{\theta_{H}+\theta_{L}}{\theta_{j}}\right)}$.

It remains to find conditions under which $Z_{i}<1$ for $i=H, L$ which guarantees that $\underline{\delta}_{i}<1$ for $i=H, L$ for some $p<1$. Note that $Z_{i}<1$ iff $\frac{\theta_{j}}{\theta_{i}}<\ln \left(\frac{\theta_{H}+\theta_{L}}{\theta_{H}}\right)+\ln \left(\frac{\theta_{H}+\theta_{L}}{\theta_{L}}\right)$. Since $\theta_{L} \leq \theta_{H}$, if $Z_{L}<1$, then $Z_{H}<1$. Thus the conjectured equilibrium exists if $Z_{L}<1$, i.e. if $\frac{\theta_{H}}{\theta_{L}}<2 \ln \left(\theta_{H}+\theta_{L}\right)-\ln \left(\theta_{H}\right)-\ln \left(\theta_{L}\right)$, which holds if $\theta_{H}$ and $\theta_{L}$ are sufficiently close.

Note that the threshold $\underline{\delta}_{i}$ is increasing in $p$, so it is easier to sustain the efficient level through these non-Markov strategies when persistence of power is lower.

\section{B3. Proof of Lemma 7 (Low-polarization equilibrium characterization)}

We proceed by first conjecturing an equilibrium strategy-payoff pair and then verifying that it satisfies guesses (G1)-(G3), equilibrium conditions (E1)-(E3), and our assumption on $\alpha^{i}$ that all proposals made on the equilibrium path are accepted when $\frac{\theta_{H}}{\theta_{L}} \leq \frac{1-\delta p}{\delta(1-p)}$ and condition $(*)$ holds.

We conjecture an equilibrium strategy-payoff pair such that for any $i, j \in\{H, L\}$ with $j \neq i$, 
the acceptance strategy $\alpha^{i}(g, \mathbf{z})$ satisfies (E1), the proposal strategies are

$$
\begin{gathered}
\gamma^{i}(g)= \begin{cases}g_{i}^{*} & \text { for } g \leq g_{i}^{*}, \\
g & \text { for } g_{i}^{*} \leq g \leq \theta_{H}+\theta_{L}, \\
\theta_{H}+\theta_{L} & \text { for } \theta_{H}+\theta_{L} \leq g,\end{cases} \\
\chi_{j}^{i}(g)= \begin{cases}0 & \text { for } g \leq \theta_{H}+\theta_{L}, \\
\frac{\theta_{j}(1-\delta p)-\theta_{i} \delta(1-p)}{(1-\delta)(1+\delta-2 \delta p)} \ln \left(\frac{g}{\theta_{H}+\theta_{L}}\right) & \text { for } \theta_{H}+\theta_{L} \leq g,\end{cases}
\end{gathered}
$$

and $\chi_{i}^{i}(g)=1-\gamma^{i}(g)-\chi_{j}^{i}(g)$, where $g_{L}^{*}=\theta_{L}$ and $g_{H}^{*}=\frac{1+\delta-2 \delta p}{1-\delta p} \theta_{H}$, and the associated payoff functions are

$$
\begin{gathered}
V_{L}(g)= \begin{cases}V_{L}^{*} & \text { for } g<g_{L}^{*}, \\
\frac{1}{1-\delta p}\left[1-g+\theta_{L} \ln (g)+\delta(1-p) W_{L}^{*}\right] & \text { for } g_{L}^{*} \leq g \leq g_{H}^{*}, \\
\frac{(1-\delta p)(1-g)}{(1+\delta-2 \delta p)(1-\delta)}+\frac{\theta_{L}}{1-\delta} \ln (g) & \begin{array}{l}
\text { for } g_{H}^{*} \leq g \leq \theta_{H}+\theta_{L} \\
C_{L} \ln (g)+D_{L}
\end{array}\end{cases} \\
W_{L}(g)= \begin{cases}W_{L}^{*} & \text { for } \theta_{H}+\theta_{L}<g, \\
\frac{1}{1-\delta p}\left[\theta_{L} \ln (g)+\delta(1-p) V_{L}(g)\right] & \text { for } g \leq g_{H}^{*},\end{cases} \\
V_{H}(g)= \begin{cases}V_{H}^{*} \\
\frac{(1-\delta p)(1-g)}{(1-\delta)(1+\delta-2 \delta p)}+\frac{\theta_{H}}{1-\delta} \ln (g), & \text { for } g<g_{H}^{*} \leq g \leq \theta_{H}^{*}+\theta_{L}, \\
C_{H} \ln (g)+D_{H} & \text { for } \theta_{H}+\theta_{L} \leq g,\end{cases} \\
W_{H}(g)= \begin{cases}W_{H}^{*} \\
\frac{1}{1-\delta p}\left[\theta_{H} \ln (g)+\delta(1-p) V_{H}(g)\right] & \text { for } g_{L}^{*} \leq g,\end{cases}
\end{gathered}
$$

where

$$
C_{i}=\frac{-(1-\delta p) \theta_{j}+\delta(1-p) \theta_{i}}{(1-\delta)(1+\delta-2 \delta p)}, D_{i}=\frac{(1-\delta p)\left(1-\theta_{L}-\theta_{H}+\left(\theta_{H}+\theta_{L}\right) \ln \left(\theta_{H}+\theta_{L}\right)\right)}{(1-\delta)(1+\delta-2 \delta p)}
$$


and

$$
\begin{aligned}
W_{L}^{*} & =\frac{\delta(1-p)}{(1+\delta-2 \delta p)(1-\delta)}\left(1-g_{H}^{*}\right)+\frac{\theta_{L}}{1-\delta} \ln \left(g_{H}^{*}\right), \\
V_{L}^{*} & =\frac{1}{1-\delta p}\left[1-\theta_{L}+\theta_{L} \ln \left(\theta_{L}\right)+\delta(1-p) W_{L}^{*}\right], \\
V_{H}^{*} & =\frac{(1-\delta p)\left(1-g_{H}^{*}\right)}{(1+\delta-2 \delta p)(1-\delta)}+\frac{\theta_{H}}{1-\delta} \ln \left(g_{H}^{*}\right), \\
W_{H}^{*} & =\frac{1}{1-\delta p}\left[\theta_{H} \ln \left(g_{L}^{*}\right)+\delta(1-p) V_{H}^{*}\right] .
\end{aligned}
$$

This conjecture satisfies (G2) and (G3). (Note that by substituting $W_{j}$ in (6), we can verify that $W_{j}(g)=K_{j}(g)$ for $g \geq g_{i}^{*}$.) So we only need to verify that (G1) is satisfied; in particular, that $g_{i}^{*} \in \arg \max f_{i}(g)$ where $f_{i}(g)=1-g+\theta_{i} \ln (g)+\delta\left[p V_{i}(g)+(1-p) W_{i}(g)\right]$.

Since $V_{i}$ and $W_{i}$ are continuous under our conjecture of the equilibrium strategy-payoff pair, $f_{i}$ is continuous. It is also piecewise differentiable. Specifically,

$$
\begin{aligned}
& f_{L}^{\prime}(g)= \begin{cases}-1+\frac{\theta_{L}}{g} & \text { for } g<g_{H}^{*}, \\
-\frac{1-\delta p}{(1-\delta)(1+\delta-2 \delta p)}+\frac{\theta_{L}}{(1-\delta) g} & \text { for } g \in\left(g_{H}^{*}, \theta_{H}+\theta_{L}\right), \\
-1+\frac{1+\delta-2 \delta p}{1-\delta p} \frac{\theta_{L}}{g}+\frac{\delta(p+\delta-2 \delta p)}{1-\delta p} \frac{C_{L}}{g} & \text { for } g>\theta_{H}+\theta_{L},\end{cases} \\
& f_{H}^{\prime}(g)= \begin{cases}-1+\frac{\theta_{H}}{g} & \text { for } g<g_{L}^{*}, \\
-1+\frac{1+\delta-2 \delta p}{1-\delta p} \frac{\theta_{H}}{g} & \text { for } g \in\left(g_{L}^{*}, g_{H}^{*}\right), \\
-\frac{1-\delta p}{(1-\delta)(1+\delta-2 \delta p)}+\frac{\theta_{H}}{(1-\delta) g} & \text { for } g \in\left(g_{H}^{*}, \theta_{H}+\theta_{L}\right), \\
-1+\frac{1+\delta-2 \delta p}{1-\delta p} \frac{\theta_{H}}{g}+\frac{\delta(p+\delta-2 \delta p)}{1-\delta p} \frac{C_{H}}{g} & \text { for } g>\theta_{H}+\theta_{L} .\end{cases}
\end{aligned}
$$

CLAIM B.1: Under our conjecture of the equilibrium strategy-payoff pair, $g_{i}^{*} \in \arg \max f_{i}(g)$ for all $i \in\{H, L\}$.

Proof: Consider $i=L$ first. Given $f_{L}$ described above, $f_{L}^{\prime}(g)>0$ if $g<g_{L}^{*}, f_{L}^{\prime}(g)=0$ if $g=g_{L}^{*}$, and $f_{L}^{\prime}(g)<0$ if $g \in\left(g_{L}^{*}, g_{H}^{*}\right)$.

Since $f_{L}^{\prime}(g)$ is decreasing for $g \in\left(g_{H}^{*}, \theta_{H}+\theta_{L}\right)$, and at $g=g_{H}^{*},-\frac{1-\delta p}{(1-\delta)(1+\delta-2 \delta p)}+\frac{\theta_{L}}{(1-\delta) g}=$ $-\frac{1-\delta p}{(1-\delta)(1+\delta-2 \delta p)}+\frac{\theta_{L}(1-\delta p)}{(1-\delta)(1+\delta-2 \delta p) \theta_{H}}<0$, it follows that $f_{L}^{\prime}(g)<0$ for $g \in\left(g_{H}^{*}, \theta_{H}+\theta_{L}\right)$.

If $\frac{(1+\delta-2 \delta p) \theta_{L}+\delta(p+\delta-2 \delta p) C_{L}}{1-\delta p} \leq 0$, then $f_{L}^{\prime}(g)<0$ for $g>\theta_{H}+\theta_{L}$. If $\frac{(1+\delta-2 \delta p) \theta_{L}+\delta(p+\delta-2 \delta p) C_{L}}{1-\delta p}>$ 0 , then $f_{L}^{\prime}(g)$ is decreasing in $g$ for $g \geq \theta_{H}+\theta_{L}$. Since at $g=\theta_{H}+\theta_{L}, f_{L}^{\prime}(g)=\frac{-(1-\delta p) \theta_{H}+(\delta-\delta p) \theta_{L}}{\left(\theta_{H}+\theta_{L}\right)(1+\delta-2 \delta p)(1-\delta)}<$ 0 , it follows that $f_{L}^{\prime}(g)<0$ for $g>\theta_{H}+\theta_{L}$.

To summarize, $f_{L}^{\prime}(g)>0$ for $g<g_{L}^{*}, f_{L}^{\prime}(g)=0$ if $g=g_{L}^{*}, f_{L}^{\prime}(g)>0$ for $g>g_{L}^{*}$, and therefore $g_{L}^{*} \in \arg \max f_{L}(g)$.

Now consider $i=H$. Given $f_{H}$ described above, $f_{H}^{\prime}(g)>0$ for $g<g_{H}^{*}$. By a similar argument as for party $L, f_{H}(g)$ is decreasing for $g>g_{H}^{*}$. Therefore $g_{H}^{*} \in \arg \max f_{H}(g)$.

Claim B.1 shows that (G1) is satisfied. We next verify that equilibrium conditions (E1)-(E3) are satisfied. Condition (E1) is satisfied by construction. 
The values $V_{L}^{*}, W_{L}^{*}, V_{H}^{*}$ and $W_{H}^{*}$ satisfy

$$
\begin{aligned}
V_{L}^{*} & =1-g_{L}^{*}+\theta_{L} \ln \left(g_{L}^{*}\right)+\delta\left[p V_{L}^{*}+(1-p) W_{L}^{*}\right], \\
W_{L}^{*} & =\theta_{L} \ln \left(g_{H}^{*}\right)+\delta\left[(1-p) V_{L}^{*}+p W_{L}^{*}\right] \\
V_{H}^{*} & =1-g_{H}^{*}+\theta_{H} \ln \left(g_{H}^{*}\right)+\delta\left[p V_{H}^{*}+(1-p) W_{H}\left(g_{H}^{*}\right)\right], \\
W_{H}^{*} & =\theta_{H} \ln \left(g_{L}^{*}\right)+\delta\left[(1-p) V_{H}^{*}+p W_{H}^{*}\right] .
\end{aligned}
$$

These together with Lemmas 1, 3 and 5 show that (E3) is satisfied, i.e., these payoff functions are consistent with the strategy profile.

The remainder of the proof shows that (E2) is satisfied. The next claim establishes that $K_{i}(g)$ is increasing in $g$, which is useful later in the proof.

CLAIM B.2: Under our conjecture of the equilibrium strategy-payoff pair, $K_{i}(g)$ is strictly increasing in $g$ for all $i \in\{H, L\}$.

Proof: Suppose $g \leq g_{L}^{*}$. Then $K_{i}(g)=\theta_{i} \ln (g)+\delta\left[(1-p) V_{i}^{*}+p W_{i}^{*}\right]$ and $K_{i}^{\prime}(g)>0$.

Suppose $g \in\left[g_{L}^{*}, g_{H}^{*}\right]$. Then $K_{L}(g)=\theta_{L} \ln (g)+\delta\left[(1-p) V_{L}(g)+p W_{L}^{*}\right]$ where $V_{L}(g)=$ $\frac{1}{1-\delta p}\left[1-g+\theta_{L} \ln (g)+\delta(1-p) W_{L}^{*}\right]$. Hence,

$$
K_{L}^{\prime}(g)=\frac{1+\delta-2 \delta p}{1-\delta p} \frac{\theta_{L}}{g}-\frac{\delta(1-p)}{1-\delta p}
$$

Since $\frac{\theta_{H}}{\theta_{L}}<\frac{1-\delta p}{\delta(1-p)}$, in the low-polarization case we have $K_{L}^{\prime}(g)>0$.

Also, since $K_{H}(g)=\theta_{H} \ln (g)+\delta(1-p) V_{H}^{*}+\delta p\left[\frac{\theta_{H}}{1-\delta p} \ln (g)+\delta(1-p) V_{H}^{*}\right]$, it follows that $K_{H}(g)$ is increasing in $g$.

Suppose $g \in\left[g_{H}^{*}, \theta_{H}+\theta_{L}\right]$. Then $K_{i}(g)=\frac{\theta_{i}}{1-\delta p} \ln (g)+\frac{\delta(1-p)}{1-\delta p} V_{i}(g)$. Substituting for $V_{i}(g)$ and taking the derivative, we get

$$
K_{i}^{\prime}(g)=\frac{1}{1-\delta}\left[\frac{-\delta(1-p)}{1+\delta-2 \delta p}+\frac{\theta_{i}}{g}\right] .
$$

Since $\frac{\theta_{H}}{\theta_{L}}<\frac{1-\delta p}{\delta(1-p)}$, it follows that $K_{i}^{\prime}(g)>0$ for $g \in\left[g_{H}^{*}, \theta_{H}+\theta_{L}\right]$.

Suppose $g \geq \theta_{H}+\theta_{L}$. Then again $K_{i}(g)=\frac{\theta_{i}}{1-\delta p} \ln (g)+\frac{\delta(1-p)}{1-\delta p} V_{i}(g)$. Substituting for $V_{i}(g)$ and taking the derivative, we get

$$
K_{i}^{\prime}(g)=\frac{\theta_{i}}{(1-\delta p) g}+\frac{\delta(1-p)}{1-\delta p} \frac{C_{i}}{g}=\frac{\theta_{i}(1-\delta p)-\theta_{j} \delta(1-p)}{(1-\delta)(1+\delta-2 \delta p) g}
$$

where $j \in\{H, L\}, j \neq i$.

For $i=H, \theta_{H}(1-\delta p)-\theta_{L} \delta(1-p)>0$ and therefore $K_{H}^{\prime}(g)>0$. For $i=L$, since $\frac{\theta_{H}}{\theta_{L}}<\frac{1-\delta p}{\delta(1-p)}$ in the low-polarization case, it follows that $\theta_{L}(1-\delta p)-\theta_{H} \delta(1-p)>0$ and therefore $K_{L}^{\prime}(g)>0$.

The claim immediately implies that the responder accepts any proposal with $g^{\prime}$ higher than the status quo $g$ and if the responder accepts a proposal with $g^{\prime}$ lower than the status quo, then the responder must receive a positive transfer. A formal statement is as follows. 
COROLLARY B.1: Consider $z^{\prime}=\left(g^{\prime}, x_{H}^{\prime}, x_{L}^{\prime}\right) \in \mathcal{B}$. For any $i \in\{H, L\}$, (i) if $g^{\prime} \geq g$, then $\alpha^{i}\left(g, \boldsymbol{z}^{\prime}\right)=1$; (ii) if $g^{\prime}<g$ and $\alpha^{i}\left(g, \boldsymbol{z}^{\prime}\right)=1$, then $x_{i}^{\prime}>0$.

For notational convenience, let $U_{i}^{P}(\mathbf{z})=x_{i}+\theta_{i} \ln (g)+\delta\left[p V_{i}(g)+(1-p) W_{i}(g)\right]$ and $U_{i}^{R}(\mathbf{z})=$ $x_{i}+\theta_{i} \ln (g)+\delta\left[(1-p) V_{i}(g)+p W_{i}(g)\right]$. That is, $U_{i}^{P}(\mathbf{z})\left(U_{i}^{R}(\mathbf{z})\right)$ denotes party $i$ 's dynamic payoff when the implemented budget is $\mathbf{z}$ in the current period and party $i$ is the proposer (responder). The next claim establishes that all equilibrium proposals are accepted.

CLAIM B.3: Under our conjecture of the equilibrium strategy-payoff pair, if condition (*) holds, then $\alpha^{j}\left(g, \pi^{i}(g)\right)=1$ for all $g$ and all $i, j \in\{H, L\}, j \neq i$.

Proof: Consider $j=H$ first.

If $g \leq g_{L}^{*}$, then $U_{H}^{R}\left(\pi^{L}(g)\right)=\theta_{H} \ln \left(g_{L}^{*}\right)+\delta\left[(1-p) V_{H}^{*}+p W_{H}^{*}\right] \geq K_{H}(g)=\theta_{H} \ln (g)+\delta[(1-$ $\left.p) V_{H}^{*}+p W_{H}^{*}\right]$ and therefore $\alpha^{H}\left(g, \pi^{L}(g)\right)=1$.

If $g \in\left[g_{L}^{*}, \theta_{H}+\theta_{L}\right]$, then $\gamma^{L}(g)=g$ and $\chi_{H}^{L}(g)=0$, which implies that $U_{H}^{R}\left(\pi^{L}(g)\right)=K_{H}(g)$ and therefore $\alpha^{H}\left(g, \pi^{L}(g)\right)=1$.

If $g>\theta_{H}+\theta_{L}$, then $\gamma^{L}(g)=\theta_{H}+\theta_{L}$ and $\chi_{H}^{L}(g)=K_{H}(g)-\theta_{H} \ln \left(\theta_{H}+\theta_{L}\right)-\delta[(1-$ p) $\left.V_{H}\left(\theta_{H}+\theta_{L}\right)+p W_{H}\left(\theta_{H}+\theta_{L}\right)\right]$. Note that $\chi_{H}^{L}(g) \leq 1-\left(\theta_{H}+\theta_{L}\right)$ if condition $(*)$ holds. With this proposal, $U_{H}^{R}\left(\pi^{L}(g)\right)=K_{H}(g)$ and therefore $\alpha^{H}\left(g, \pi^{L}(g)\right)=1$.

Now consider $j=L$.

If $g \leq g_{H}^{*}$, then $U_{L}^{R}\left(\pi^{H}(g)\right)=\theta_{L} \ln \left(g_{H}^{*}\right)+\delta\left[(1-p) V_{L}\left(g_{H}^{*}\right)+p W_{L}\left(g_{H}^{*}\right)\right]$. Since $K_{L}^{\prime}(g)>0$ by Claim B.2 and $U_{L}^{R}\left(\pi^{H}(g)\right)=K_{L}\left(g_{H}^{*}\right)$, it follows that $U_{L}^{R}\left(\pi^{H}(g)\right) \geq K_{L}(g)$ and therefore $\alpha^{L}\left(g, \pi^{H}(g)\right)=1$ for $g \leq g_{H}^{*}$.

If $g \geq g_{H}^{*}$, then an argument similar to the case of $j=H$ shows that $U_{L}^{R}\left(\pi^{H}(g)\right)=K_{L}(g)$ and therefore $\alpha^{L}\left(g, \pi^{H}(g)\right)=1$.

We next show that the proposer has no profitable one-shot deviation. Consider the following three cases for party $L$.

- $g \leq g_{L}^{*}$ : Since $g_{L}^{*}=\arg \max f_{L}(g)$, party $L$ has no incentive to deviate from proposing $\gamma^{L}(g)=g_{L}^{*}$ and $\chi_{H}^{L}(g)=0$.

- $g_{L}^{*}<g \leq \theta_{H}+\theta_{L}$ : We first show that proposing $\pi^{L}(g)$ is better than proposing $\left(\hat{g}, \hat{x}_{H}, \hat{x}_{L}\right)$ with $\hat{g}>g$ and then show that it is better than proposing $\left(\hat{g}, \hat{x}_{H}, \hat{x}_{L}\right)$ with $\hat{g}<g$.

$-\hat{g}>g$ : Consider $\hat{\mathbf{z}}=(\hat{g}, 0,1-\hat{g})$. Then $U_{L}^{P}(\hat{\mathbf{z}})=f_{L}(\hat{g})$. As shown in the proof of Claim B.1, $f_{L}(\hat{g})$ is decreasing for $\hat{g}>g_{L}^{*}$. Since $\pi^{L}(g)=(g, 0,1-g)$, this implies that $U_{L}^{P}\left(\pi^{L}(g)\right)>U_{L}^{P}(\hat{\mathbf{z}})$ for any $\hat{g}>g>g_{L}^{*}$. Since party $L$ 's payoff is decreasing in $x_{H}, U_{L}^{P}(\hat{\mathbf{z}}) \geq U_{L}^{P}\left(\left(\hat{g}, \hat{x}_{H}, \hat{x}_{L}\right)\right)$ for any $\left(\hat{g}, \hat{x}_{H}, \hat{x}_{L}\right) \in \mathcal{B}$, it follows that $U_{L}^{P}\left(\pi^{L}(g)\right)>U_{L}^{P}\left(\left(\hat{g}, \hat{x}_{H}, \hat{x}_{L}\right)\right)$ for any $\hat{g}>g>g_{L}^{*}$. Also, since $\alpha^{H}\left(g, \pi^{L}(g)\right)=1$ by Claim B.3, and $U_{L}^{P}\left(\pi^{L}(g)\right)>U_{L}^{P}((g, 0,0))$, the status quo payoff, it follows that proposing $\pi^{L}(g)$ is better than proposing any $\left(\hat{g}, \hat{x}_{H}, \hat{x}_{L}\right) \in \mathcal{B}$ with $\hat{g}>g$.

$-\hat{g}<g$ : If $\hat{g}<g$, then by Corollary B.1, $\alpha^{H}\left(g,\left(\hat{g}, \hat{x}_{H}, \hat{x}_{L}\right)\right)=1$ only if $\hat{x}_{H}>0$. Since party $L$ 's payoff is strictly decreasing in $x_{H}$, we only need to consider proposals 
such that the responder's acceptance constraint (5) is binding. From (5),

$$
\hat{x}_{H}=K_{H}(g)-\theta_{H} \ln (\hat{g})-\delta\left[(1-p) V_{H}(\hat{g})+p W_{H}(\hat{g})\right] .
$$

Consider $\hat{\mathbf{z}}=\left(\hat{g}, \hat{x}_{H}, \hat{x}_{L}\right)$ such that (B4) holds. Substituting for $\hat{x}_{H}$ from (B4) and taking the derivative, we get

(B5)

$$
\frac{\partial U_{L}^{P}}{\partial \hat{g}}=-1+\frac{\theta_{H}+\theta_{L}}{\hat{g}}+\delta\left[(1-p) V_{H}^{\prime}(\hat{g})+p W_{H}^{\prime}(\hat{g})\right]+\delta\left[p V_{L}^{\prime}(\hat{g})+(1-p) W_{L}^{\prime}(\hat{g})\right]
$$

For $\hat{g}<g_{L}^{*}, \frac{\partial U_{L}^{P}}{\partial \hat{g}}=-1+\frac{\theta_{H}+\theta_{L}}{\hat{g}}>0$.

For $g_{L}^{*}<\hat{g}<g_{H}^{*}, \frac{\partial U_{L}^{P}}{\partial \hat{g}}=-1+\frac{\theta_{H}+\theta_{L}}{\hat{g}}+\frac{\delta p}{1-\delta p} \frac{\theta_{H}}{\hat{g}}+\frac{\delta p}{1-\delta p}\left(-1+\frac{\theta_{L}}{g}\right)=\frac{1}{1-\delta p}(-1+$ $\left.\frac{\theta_{H}+\theta_{L}}{\hat{g}}\right)>0$.

For $g_{H}^{*}<\hat{g}<g \leq \theta_{H}+\theta_{L}, \frac{\partial U_{L}^{P}}{\partial \hat{g}}=-1+\frac{1+\delta-2 \delta p}{1-\delta p} \frac{\theta_{L}}{\hat{g}}+\frac{\delta(p+\delta-2 \delta p)}{1-\delta p} V_{L}^{\prime}(\hat{g})+\frac{1}{1-\delta p} \frac{\theta_{H}}{\hat{g}}+$ $\frac{\delta(1-p)}{1-\delta p} V_{H}^{\prime}(\hat{g})=\frac{1}{1-\delta}\left(-1+\frac{\theta_{H}+\theta_{L}}{g}\right)>0$.

So $U_{L}^{P}(\hat{\mathbf{z}})$ is increasing in $\hat{g}$ for $\hat{g}<g$, and therefore the proposer has no incentive to make any proposal with $\hat{g}<g$.

- $g>\theta_{H}+\theta_{L}$ : Consider $\hat{\mathbf{z}}=(\hat{g}, 0,1-\hat{g})$ with $\hat{g}>g$. By Corollary B.1, $\alpha^{H}(g, \hat{\mathbf{z}})=1$. Since $U_{L}^{P}(\hat{\mathbf{z}})=f_{L}(\hat{g})$ and $f_{L}(\hat{g})$ is decreasing in $\hat{g}$ for $\hat{g}>g>\theta_{H}+\theta_{L}$, it follows that $U_{L}^{P}((g, 0,1-g)) \geq U_{L}^{P}((\hat{g}, 0,1-\hat{g}))$ if $\hat{g} \geq g$.

Now consider $\hat{\mathbf{z}}=\left(\hat{g}, \hat{x}_{H}, \hat{x}_{L}\right)$ such that $\hat{g} \leq g$ and $\alpha^{H}(g, \hat{\mathbf{z}})=1$. By Corollary B.1, $\hat{x}_{H}>$ 0 if $\hat{g}>g$. Again we only need to consider proposals such that the acceptance constraint binds. As before, we obtain (B5). Substituting for $V_{L}^{\prime}(\hat{g}), W_{L}^{\prime}(\hat{g}), V_{H}^{\prime}(\hat{g}), W_{H}^{\prime}(\hat{g})$, we get

$$
\frac{\partial U_{L}^{P}}{\partial \hat{g}}=-1+\frac{1+\delta-2 \delta p}{1-\delta p} \frac{\theta_{L}}{\hat{g}}+\frac{\delta(p+\delta-2 \delta p)}{1-\delta p} \frac{C_{L}}{\hat{g}}+\left(\frac{1}{1-\delta p} \frac{\theta_{H}}{\hat{g}}+\frac{\delta(1-p)}{1-\delta p} \frac{C_{H}}{\hat{g}}\right)=-1+\frac{\theta_{H}+\theta_{L}}{\hat{g}} .
$$

Since $\gamma^{L}(g)=\theta_{H}+\theta_{L}$, it follows that $U_{L}^{P}\left(\pi^{L}(g)\right) \geq U_{L}^{P}(\hat{\mathbf{z}})$ for any $\hat{\mathbf{z}}=\left(\hat{g}, \hat{x}_{H}, \hat{x}_{L}\right)$ such that $\hat{g}<g$ and $\alpha^{H}(g, \hat{\mathbf{z}})=1$. Combined with $U_{L}^{P}((g, 0,1-g)) \geq U_{L}^{P}((\hat{g}, 0,1-\hat{g}))$ if $\hat{g} \geq g, \pi^{L}(g)$ is optimal for party $L$ to propose.

Party $H$ also has no incentive to deviate. We omit the details of the proof because the argument is similar to that for party $L$.

\section{B4. Low-polarization equilibrium when condition (*) fails:}

Denote by $\mathbf{z}_{j}^{e}$ the proposal $\left(\theta_{H}+\theta_{L}, x_{H}, x_{L}\right)$ where $x_{i}=0$ and $x_{j}=1-\theta_{H}-\theta_{L}$. Recall that in Lemma 7, we assume condition $(*)$ holds, which ensures the responder $j$ accepts the proposal $\mathbf{z}_{j}^{e}$ even when the status quo $g$ is high. What happens if condition (*) fails, that is, if $\alpha^{j}\left(g, \mathbf{z}_{j}^{e}\right)=0$ for $g$ sufficiently high? Then, instead of proposing $g^{\prime}=\theta_{H}+\theta_{L}$, party $i$ 
proposes $g^{\prime}>\theta_{H}+\theta_{L}, x_{i}^{\prime}=0$, and $x_{j}^{\prime}=1-g^{\prime}$ such that party $j$ is just willing to accept. Figure B1 illustrates the parties' proposal strategies when condition $(*)$ fails. In the figure (G1)-(G2) are still satisfied, but for very high status quos, (G3) is violated. As shown in Section V.B, the failure of condition $(*)$ does not affect the set of steady states.

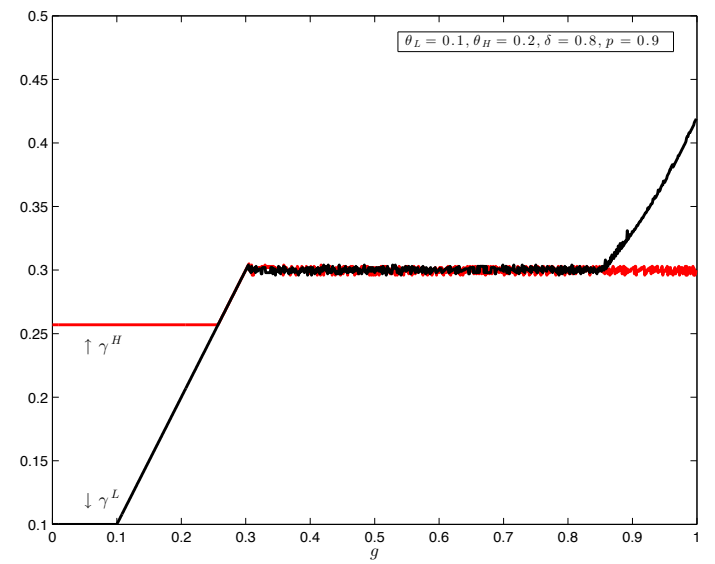

Figure B1. : $\gamma^{i}(g)$ in low-polarization case when $(*)$ fails

\section{B5. Proof of Proposition 4}

We formally characterize an equilibrium with the properties given in Propostion 4 in the high-polarization case where $\frac{\theta_{H}}{\theta_{L}}>\frac{1-\delta p}{\delta(1-p)}$. Recall that $f_{i}(g)$ defined in (8) is party $i$ 's dynamic payoff when the public spending in the current period is $g$ and party $i$ receives the remaining surplus. Motivated by Figure 5, we make the following guesses about an equilibrium strategypayoff pair.

$\left(\mathrm{G1}^{\prime}\right)$ There exist $g_{L}^{*}$ and $g_{H}^{*}$ with $g_{L}^{*}<\theta_{H}+\theta_{L}<g_{H}^{*}$ such that $g_{i}^{*} \in \arg \max f_{i}(g)$ for $i \in\{H, L\}$.

$\left(\mathrm{G} 2^{\prime}\right)$ If $g \leq g_{L}^{*}$, then $\pi^{L}(g)=\pi^{L}\left(g_{L}^{*}\right)$ and specifically $\gamma^{L}(g)=g_{L}^{*}$; if $g \in\left[g_{L}^{*}, \theta_{H}+\theta_{L}\right]$, then $\gamma^{L}(g)=g$; if $g \geq \theta_{H}+\theta_{L}$, then $\gamma^{L}(g)=\theta_{H}+\theta_{L}$. If $g \geq g_{L}^{*}$, then $W_{H}(g)=K_{H}(g)$.

$\left(\mathrm{G}^{\prime}\right)$ There exist $\underline{g}_{H}$ and $\tilde{g}_{H}$ that satisfy $g_{L}^{*} \leq \underline{g}_{H}<\tilde{g}_{H}<\theta_{H}+\theta_{L}$ such that (i) $\pi^{H}(g)=$ $\pi^{H}\left(g_{H}^{*}\right)$ for $g \leq \underline{g}_{H}$ and $g \geq g_{H}^{*}$; (ii) if $g \in\left[\underline{g}_{H}, g_{H}^{*}\right]$ then $W_{L}(g)=K_{L}(g)$; (iii) if $g \leq \tilde{g}_{H}$ or if $g \geq \theta_{H}+\theta_{L}$, then $\chi_{L}^{H}(g)=0$; and (iv)

$$
\gamma^{H}(g)= \begin{cases}g_{H}^{*} & \text { for } g \leq g_{H} \\ g^{\prime} \in\left[\theta_{H}+\theta_{L}, g_{H}^{*}\right] & \text { for } \underline{g}_{H} \leq g \leq \tilde{g}_{H} \\ \theta_{H}+\theta_{L} & \text { for } \tilde{g}_{H} \leq g \leq \theta_{H}+\theta_{L} \\ g & \text { for } \theta_{H}+\theta_{L} \leq g \leq g_{H}^{*} \\ g_{H}^{*} & \text { for } g_{H}^{*} \leq g\end{cases}
$$


where $g^{\prime}$ is a function of $g$ satisfying $\theta_{L} \ln \left(g^{\prime}\right)+\delta\left[(1-p) V_{L}\left(g^{\prime}\right)+p W_{L}\left(g^{\prime}\right)\right]=K_{L}(g)$.

$\left(\mathrm{G} 4^{\prime}\right)$ If $\gamma^{i}(g)=\theta_{H}+\theta_{L}$, then $V_{i}(g)$ is piecewise linear in $g$ and $\ln (g)$.

We first establish some properties of an equilibrium strategy-payoff pair that satisfies $\left(\mathrm{G}^{\prime}\right)$ $\left(\mathrm{G} 4^{\prime}\right)$. Suppose $\sigma=\left(\left(\pi^{H}, \alpha^{H}\right),\left(\pi^{L}, \alpha^{L}\right)\right)$ and $\left(V_{H}, W_{H}, V_{L}, W_{L}\right)$ is an equilibrium strategypayoff pair that satisfies $\left(\mathrm{G} 1^{\prime}\right)-\left(\mathrm{G} 4^{\prime}\right)$. Recall that $V_{i}^{*}=\max _{g} f_{i}(g)$ is proposer $i$ 's highest payoff without the responder's constraint (5). As in the low-polarization case, we denote $W_{L}\left(g_{H}^{*}\right)$ by $W_{L}^{*}$ and $W_{H}\left(g_{L}^{*}\right)$ by $W_{H}^{*}$.

LEMMA B.1: Under $\left(G 1^{\prime}\right)$ and $\left(G \mathscr{2}^{\prime}\right)$, if $g \leq g_{L}^{*}$, then $V_{L}(g)=V_{L}^{*}, \chi_{L}^{L}(g)=1-g_{L}^{*}, \chi_{H}^{L}(g)=$ 0 , and $W_{H}(g)=W_{H}^{*}$. Under $\left(G 3^{\prime}\right)$, if $g \leq \underline{g}_{H}$ or $g \geq g_{H}^{*}$, then $V_{H}(g)=V_{H}^{*}, \chi_{H}^{H}(g)=1-g_{H}^{*}$, $\chi_{L}^{H}(g)=0$, and $W_{L}(g)=W_{L}^{*}$.

We omit the proof since it is similar to that of Lemma 1.

LEMMA B.2: Under $\left(G 1^{\prime}\right)-\left(G 3^{\prime}\right)$, (i) if $g \in\left[g_{L}^{*}, \underline{g}_{H}\right]$, then

$$
V_{L}(g)=\frac{1}{1-\delta p}\left[1-g+\theta_{L} \ln (g)+\delta(1-p) W_{L}^{*}\right],
$$

(ii) if $g \in\left[\underline{g}_{H}, \theta_{H}+\theta_{L}\right]$, then

$$
V_{L}(g)=\frac{(1-\delta p)(1-g)}{(1-\delta)(1+\delta-2 \delta p)}+\frac{\theta_{L}}{1-\delta} \ln (g),
$$

and (iii) if $g \in\left[\theta_{H}+\theta_{L}, g_{H}^{*}\right]$, then

$$
V_{H}(g)=\frac{(1-\delta p)(1-g)}{(1-\delta)(1+\delta-2 \delta p)}+\frac{\theta_{H}}{1-\delta} \ln (g) .
$$

We omit the proof since it is similar to that of Lemma 3.

To simplify notation, define

$$
\theta_{i}^{*} \equiv \frac{1+\delta-2 \delta p}{1-\delta p} \theta_{i}
$$

LEMMA B.3: $\operatorname{Under}\left(G 1^{\prime}\right)-\left(G 3^{\prime}\right), g_{L}^{*}=\theta_{L}$ and $g_{H}^{*}=\theta_{H}^{*}$.

Proof: We omit the proof for $g_{L}^{*}$ since it is the same as that of Lemma 4.

Now consider $g_{H}^{*}$. If $g>g_{H}^{*}$, then $V_{H}^{\prime}(g)=0$ by Lemma B.1 and $W_{H}^{\prime}(g)=\frac{\theta_{H}}{(1-\delta p) g}$ by Lemma 2, and therefore

$$
f_{H}^{\prime}(g)=-1+\frac{\theta_{H}}{g}+\delta(1-p) W_{H}^{\prime}(g)=-1+\frac{\theta_{H}^{*}}{g} .
$$

If $g_{H}^{*}<\theta_{H}^{*}$, then (B8) implies that $f_{H}^{\prime}(g)>0$ for $g \in\left(g_{H}^{*}, \theta_{H}^{*}\right)$, contradicting that $g_{H}^{*} \in$ $\arg \max f_{H}(g)$. Hence $g_{H}^{*} \geq \theta_{H}^{*}$.

If $g \in\left(\theta_{H}+\theta_{L}, g_{H}^{*}\right)$, then by $\left(\mathrm{G} 3^{\prime}\right), f_{H}(g)=V_{H}(g)$, and by $(\mathrm{B} 7)$

$$
f_{H}^{\prime}(g)=-\frac{1-\delta p}{(1-\delta)(1+\delta-2 \delta p)}+\frac{\theta_{H}}{(1-\delta) g} .
$$


If $g_{H}^{*}>\theta_{H}^{*}$, then (B9) implies that $f_{H}^{\prime}(g)<0$ for $g \in\left(\theta_{H}^{*}, g_{H}^{*}\right)$, contradicting that $g_{H}^{*} \in$ $\arg \max f_{H}(g)$. Hence $g_{H}^{*} \leq \theta_{H}^{*}$.

Since $g_{H}^{*} \leq \theta_{H}^{*}$ and $g_{H}^{*} \geq \theta_{H}^{*}$, it follows that $g_{H}^{*}=\theta_{H}^{*}$.

Recall that we guess in $\left(\mathrm{G} 4^{\prime}\right)$ that $V_{i}$ is piecewise linear in $g$ and $\ln (g)$ if $\gamma^{i}(g)=\theta_{H}+\theta_{L}$. Specifically, suppose that for $g \in\left[\theta_{H}+\theta_{L}, g_{H}^{*}\right], V_{L}(g)$ takes the form $V_{L}(g)=B_{L}^{1} g+C_{L}^{1} \ln (g)+$ $D_{L}^{1}$; for $g \geq g_{H}^{*}$ such that $\gamma_{L}(g)=\theta_{H}+\theta_{L}, V_{L}(g)$ takes the form $V_{L}(g)=B_{L}^{2} g+C_{L}^{2} \ln (g)+D_{L}^{2}$; for $g \in\left[\tilde{g}_{H}, \theta_{H}+\theta_{L}\right], V_{H}(g)$ takes the form $V_{H}(g)=B_{H}^{1} g+C_{H}^{1} \ln (g)+D_{H}^{1}$.

LEMMA B.4: Under $\left(G 1^{\prime}\right)-\left(G 4^{\prime}\right), B_{i}^{1}=\frac{\delta(1-p)(1-\delta p)}{(1-\delta)(1+\delta-2 \delta p)}$ and $C_{i}^{1}=-\frac{\theta_{j}}{1-\delta}$ for $i, j \in\{H, L\}$ with $j \neq i$, and $B_{L}^{2}=0, C_{L}^{2}=-\frac{\theta_{H}}{1-\delta p}$.

Proof: Similar to the proof of Lemma 5, we can write

$$
V_{i}(g)=\chi_{i}^{i}(g)+\frac{1+\delta-2 \delta p}{1-\delta p} \theta_{i} \ln \left(\theta_{H}+\theta_{L}\right)+\frac{\delta(p+\delta-2 \delta p)}{1-\delta p} V_{i}\left(\theta_{H}+\theta_{L}\right)
$$

where

$$
\begin{aligned}
\chi_{j}^{i}(g) & =K_{j}(g)-\frac{\theta_{j}}{1-\delta p} \ln \left(\theta_{H}+\theta_{L}\right)-\frac{\delta(1-p)}{1-\delta p} V_{j}\left(\theta_{H}+\theta_{L}\right), \\
K_{j}(g) & =\frac{\theta_{j}}{1-\delta p} \ln (g)+\frac{\delta(1-p)}{1-\delta p} V_{j}(g) .
\end{aligned}
$$

If $g \in\left[\theta_{H}+\theta_{L}, g_{H}^{*}\right]$, then $V_{H}(g)=\frac{(1-\delta p)(1-g)}{(1-\delta)(1+\delta-2 \delta p)}+\frac{\theta_{H}}{1-\delta} \ln (g)$ by Lemma B.2. Substituting in $K_{H}(g)$, we get

$$
K_{H}(g)=\frac{\delta(1-p)(1-g)}{(1-\delta)(1+\delta-2 \delta p)}+\frac{\theta_{H}}{1-\delta} \ln (g) .
$$

Substituting in $V_{L}(g)$ and matching coefficients, we get $B_{L}^{1}=\frac{\delta(1-p)}{(1-\delta)(1+\delta-2 \delta p)}$ and $C_{L}^{1}=-\frac{\theta_{H}}{1-\delta}$. A similar argument shows that $B_{H}^{1}=\frac{\delta(1-p)}{(1-\delta)(1+\delta-2 \delta p)}$ and $C_{H}^{1}=-\frac{\theta_{L}}{1-\delta}$.

To find $B_{L}^{2}$ and $C_{L}^{2}$, note that if $g \geq g_{H}^{*}$, then by Lemma B.1, $V_{H}(g)=V_{H}^{*}$. By Lemma 2, $K_{H}(g)=\frac{\theta_{H}}{1-\delta p} \ln (g)+\frac{\delta(1-p)}{1-\delta p} V_{H}^{*}$. Matching coefficients gives $B_{L}^{2}=0$ and $C_{L}^{2}=-\frac{\theta_{H}}{1-\delta p}$.

We next find the thresholds $\underline{g}_{H}$ and $\tilde{g}_{H}$ that are consistent with $\left(\mathrm{G}^{\prime}\right)-\left(\mathrm{G} 4^{\prime}\right)$.

LEMMA B.5: Under $\left(G 1^{\prime}\right)-\left(G 4^{\prime}\right)$, the threshold $\underline{g}_{H} \in\left(0, \theta_{H}+\theta_{L}\right)$ is given by $\underline{g}_{H}=\psi$ where

$$
\begin{aligned}
\psi=\min \{g \geq 0 & : \frac{\delta(1-p)(1-g)}{(1-\delta)(1+\delta-2 \delta p)}+\frac{\theta_{L}}{1-\delta} \ln (g) \\
= & \frac{\delta(1-p)}{(1-\delta)(1+\delta-2 \delta p)}+\frac{1}{1-\delta p}\left[\theta_{L}-\frac{\delta(1-p)}{1-\delta} \theta_{H}\right] \ln \left(\theta_{H}^{*}\right) \\
& \left.+\frac{\delta(1-p)}{(1-\delta p)(1-\delta)}\left[\left(\theta_{H}+\theta_{L}\right)\left[\ln \left(\theta_{H}+\theta_{L}\right)-1\right]+\frac{\delta(1-p)}{1+\delta-2 \delta p} \theta_{H}^{*}\right]\right\},
\end{aligned}
$$

and $\psi$ is a decreasing function of $\theta_{H}$. 
Proof: By $\left(\mathrm{G} 3^{\prime}\right)\left(\right.$ ii) and (iv), the threshold $\underline{g}_{H}$ satisfies

$$
\theta_{L} \ln \left(g_{H}^{*}\right)+\delta\left[(1-p) V_{L}\left(g_{H}^{*}\right)+p W_{L}\left(g_{H}^{*}\right)\right]=W_{L}\left(\underline{g}_{H}\right)=K_{L}\left(\underline{g}_{H}\right) .
$$

$\operatorname{By}\left(\mathrm{G} 3^{\prime}\right)(\mathrm{ii}), W_{L}\left(g_{H}^{*}\right)=K_{L}\left(g_{H}^{*}\right)$. Hence by Lemma 2, we can rewrite the left-hand side of the above equation as

$$
\frac{\theta_{L}}{1-\delta p} \ln \left(g_{H}^{*}\right)+\frac{\delta(1-p)}{1-\delta p} V_{L}\left(g_{H}^{*}\right)
$$

By $\left(\mathrm{G} 1^{\prime}\right), g_{H}^{*}>\theta_{H}+\theta_{L}$. Hence $\gamma^{L}\left(g_{H}^{*}\right)=\theta_{H}+\theta_{L}$ by $\left(\mathrm{G} 2^{\prime}\right)$. So $V_{L}\left(g_{H}^{*}\right)$ can be written as

$$
V_{L}\left(g_{H}^{*}\right)=\chi_{L}^{L}\left(g_{H}^{*}\right)+\frac{1+\delta-2 \delta p}{1-\delta p} \theta_{L} \ln \left(\theta_{H}+\theta_{L}\right)+\frac{\delta(p+\delta-2 \delta p)}{1-\delta p} V_{L}\left(\theta_{H}+\theta_{L}\right),
$$

where $\chi_{L}^{L}\left(g_{H}^{*}\right)=1-\chi_{H}^{L}\left(g_{H}^{*}\right)-\gamma^{L}\left(g_{H}^{*}\right)=1-\chi_{H}^{L}\left(g_{H}^{*}\right)-\theta_{H}-\theta_{L}$, and

$$
\begin{aligned}
& \chi_{H}^{L}\left(g_{H}^{*}\right)=K_{H}\left(g_{H}^{*}\right)-\frac{\theta_{H}}{1-\delta p} \ln \left(\theta_{H}+\theta_{L}\right)-\frac{\delta(1-p)}{1-\delta p} V_{H}\left(\theta_{H}+\theta_{L}\right), \\
& K_{H}\left(g_{H}^{*}\right)=\frac{\theta_{H}}{1-\delta p} \ln \left(g_{H}^{*}\right)+\frac{\delta(1-p)}{1-\delta p} V_{H}\left(g_{H}^{*}\right) .
\end{aligned}
$$

By Lemma B.2,

$V_{i}\left(\theta_{H}+\theta_{L}\right)=\frac{(1-\delta p)\left(1-\theta_{H}-\theta_{L}\right)}{(1-\delta)(1+\delta-2 \delta p)}+\frac{\theta_{i}}{1-\delta} \ln \left(\theta_{H}+\theta_{L}\right), \quad V_{H}\left(g_{H}^{*}\right)=\frac{(1-\delta p)\left(1-g_{H}^{*}\right)}{(1-\delta)(1+\delta-2 \delta p)}+\frac{\theta_{H}}{1-\delta} \ln \left(g_{H}^{*}\right)$.

Substituting in all expressions, (B12) becomes

$$
\frac{\delta(1-p)}{(1-\delta)(1+\delta-2 \delta p)}+\frac{1}{1-\delta p}\left[\theta_{L}-\frac{\delta(1-p)}{1-\delta} \theta_{H}\right] \ln \left(g_{H}^{*}\right)+\frac{\delta(1-p)}{(1-\delta p)(1-\delta)}\left[\left(\theta_{H}+\theta_{L}\right)\left[\ln \left(\theta_{H}+\theta_{L}\right)-1\right]+\frac{\delta(1-p)}{1+\delta-2 \delta p} g_{H}^{*}\right] .
$$

By $\left(\mathrm{G} 3^{\prime}\right)(\mathrm{ii})$ and Lemma 2 , we can write $K_{L}\left(\underline{g}_{H}\right)$ as

$$
K_{L}\left(\underline{g}_{H}\right)=\frac{\theta_{L}}{1-\delta p} \ln \left(\underline{g}_{H}\right)+\frac{\delta(1-p)}{1-\delta p} V_{L}\left(\underline{g}_{H}\right) .
$$

By Lemma B.2 this becomes

$$
K_{L}\left(\underline{g}_{H}\right)=\frac{\theta_{L}}{1-\delta} \ln \left(\underline{g}_{H}\right)+\frac{\delta(1-p)}{(1-\delta)(1+\delta-2 \delta p)}\left(1-\underline{g}_{H}\right) .
$$

By Lemma B.3 $g_{H}^{*}=\theta_{H}^{*}$, hence $\underline{g}_{H}$ is given by

$$
\begin{aligned}
\frac{\theta_{L}}{1-\delta} \ln \left(\underline{g}_{H}\right)+ & \frac{\delta(1-p)}{(1-\delta)(1+\delta-2 \delta p)}\left(1-\underline{g}_{H}\right)= \\
& \frac{\delta(1-p)}{(1-\delta)(1+\delta-2 \delta p)}+\frac{1}{1-\delta p}\left[\theta_{L}-\frac{\delta(1-p)}{1-\delta} \theta_{H}\right] \ln \left(\theta_{H}^{*}\right) \\
& +\frac{\delta(1-p)}{(1-\delta p)(1-\delta)}\left[\left(\theta_{H}+\theta_{L}\right)\left[\ln \left(\theta_{H}+\theta_{L}\right)-1\right]+\frac{\delta(1-p)}{1+\delta-2 \delta p} \theta_{H}^{*}\right] .
\end{aligned}
$$


Let $l(x)=\frac{\theta_{L}}{1-\delta} \ln (x)+\frac{\delta(1-p)}{(1-\delta)(1+\delta-2 \delta p)}(1-x)$, and denote the right-hand side of (B13) by $R$. At most two values of $\underline{g}_{H}$ satisfy (B13) since $l(x)$ is strictly concave. We show below only one is lower than $\theta_{H}+\theta_{L}$ and hence it is a candidate for $\underline{g}_{H}$ by $\left(\mathrm{G} 3^{\prime}\right)$. Note that

$$
\begin{aligned}
l\left(\theta_{H}+\theta_{L}\right)-R=\quad & \frac{\theta_{L}(1-\delta)-\theta_{H} \delta(1-p)}{(1-\delta p)(1-\delta)} \ln \left(\theta_{H}+\theta_{L}\right)+\frac{\delta^{2}(1-p)^{2}}{(1-\delta)(1+\delta-2 \delta p)(1-\delta p)}\left(\theta_{H}+\theta_{L}\right) \\
& -\left[\frac{\theta_{L}(1-\delta)-\theta_{H} \delta(1-p)}{(1-\delta p)(1-\delta))} \ln \left(\theta_{H}^{*}\right)+\frac{\delta^{2}(1-p)^{2}}{(1-\delta)(1+\delta-2 \delta p)(1-\delta p)}\left(\theta_{H}^{*}\right)\right] .
\end{aligned}
$$

Define $h(x)=\frac{\theta_{L}(1-\delta)-\theta_{H} \delta(1-p)}{(1-\delta p)(1-\delta)} \ln (x)+\frac{\delta^{2}(1-p)^{2}}{(1-\delta)(1+\delta-2 \delta p)(1-\delta p)} x$, then $l\left(\theta_{H}+\theta_{L}\right)-R=h\left(\theta_{H}+\right.$ $\left.\theta_{L}\right)-h\left(\theta_{H}^{*}\right)$. It is straightforward to show $h^{\prime}(x)<0$. Since $\theta_{H}+\theta_{L}<\theta_{H}^{*}$, it follows $l\left(\theta_{H}+\theta_{L}\right)-R>0$. Given $l\left(\theta_{H}+\theta_{L}\right)-R>0$, the value that satisfies (B13) such that $\underline{g}_{H}<\theta_{H}+\theta_{L}$ must be the minimum of the solutions to (B13).

At $\psi, l(x)$ is strictly increasing, and it is straightforward to show that $R$ is decreasing in $\theta_{H}$ in the high-polarization case. Hence $\psi$ is decreasing in $\theta_{H}$.

LEMMA B.6: Under $\left(G 1^{\prime}\right)-\left(G 4^{\prime}\right)$, the threshold $\tilde{g}_{H} \in\left(0, \theta_{H}+\theta_{L}\right)$ is given by

$$
\frac{\delta(1-p)\left(1-\tilde{g}_{H}\right)}{(1-\delta)(1+\delta-2 \delta p)}+\frac{\theta_{L}}{1-\delta} \ln \left(\tilde{g}_{H}\right)=\frac{\delta(1-p)\left(1-\theta_{L}-\theta_{H}\right)}{(1-\delta)(1+\delta-2 \delta p)}+\frac{\theta_{L}}{1-\delta} \ln \left(\theta_{H}+\theta_{L}\right) .
$$

Proof: By $\left(\mathrm{G} 3^{\prime}\right)$ (ii) and (iv), the threshold $\tilde{g}_{H}$ satisfies

$$
\theta_{L} \ln \left(\theta_{H}+\theta_{L}\right)+\delta\left[(1-p) V_{L}\left(\theta_{H}+\theta_{L}\right)+p W_{L}\left(\theta_{H}+\theta_{L}\right)\right]=K_{L}\left(\tilde{g}_{H}\right) .
$$

By Lemma B.2, $V_{L}(g)=\frac{(1-\delta p)(1-g)}{(1-\delta)(1+\delta-2 \delta p)}+\frac{\theta_{L}}{1-\delta} \ln (g)$ for $g \in\left[\tilde{g}_{H}, \theta_{H}+\theta_{L}\right]$. Substituting this in (B16) and using Lemma 2, we get (B15).

In $\left(\mathrm{G}^{\prime}\right)$, we guess that $\gamma^{L}(g)=\theta_{H}+\theta_{L}$ for all $g \geq \theta_{H}+\theta_{L}$. This is analogous to the low-polarization case and we need a condition similar to $(*)$ to guarantee that it holds in equilibrium.

LEMMA B.7: Under $\left(G 1^{\prime}\right)-\left(G 4^{\prime}\right)$, if

$$
1-\left(\theta_{H}+\theta_{L}\right)+\frac{\theta_{H}}{1-\delta} \ln \left(\theta_{H}+\theta_{L}\right) \geq \frac{\delta(1-p)\left(\theta_{H}+\theta_{L}-\theta_{H}^{*}\right)}{(1-\delta)(1+\delta-2 \delta p)}+\frac{\delta(1-p) \theta_{H}}{(1-\delta p)(1-\delta)} \ln \left(\theta_{H}^{*}\right) .
$$

then $\alpha^{H}\left(g,\left(\theta_{H}+\theta_{L}, x_{H}, x_{L}\right)\right)=1$ with $x_{H}=1-\theta_{H}-\theta_{L}, x_{L}=0$.

Proof: For any $g \geq \theta_{H}+\theta_{L}$, we have $\alpha^{H}\left(g,\left(\theta_{H}+\theta_{L}, x_{H}, x_{L}\right)\right)=1$ with $x_{H}=1-\theta_{H}-\theta_{L}$, $x_{L}=0$ if

$$
1-\left(\theta_{H}+\theta_{L}\right)+\theta_{H} \ln \left(\theta_{H}+\theta_{L}\right)+\delta\left[(1-p) V_{H}\left(\theta_{H}+\theta_{L}\right)+p W_{H}\left(\theta_{H}+\theta_{L}\right)\right] \geq K_{H}(g) .
$$


Substituting for $K_{H}(g)$ and $W_{H}(g)$ using Lemma 2 and substituting for $V_{H}(g)=V_{H}^{*}$ for $g \geq g_{H}^{*}$ using Lemma B.1, the inequality becomes

$$
1-\left(\theta_{H}+\theta_{L}\right)+\frac{\theta_{H}}{1-\delta p} \ln \left(\theta_{H}+\theta_{L}\right)+\frac{\delta(1-p)}{1-\delta p} V_{H}\left(\theta_{H}+\theta_{L}\right) \geq \frac{\theta_{H}}{1-\delta p} \ln (g)+\frac{\delta(1-p)}{1-\delta p} V_{H}^{*} .
$$

Note that the right-hand side of (B17) is increasing in $g$, implying that if the inequality holds for $g=1$, then it holds for all $g \geq \theta_{H}+\theta_{L}$. Substituting for $V_{H}\left(\theta_{H}+\theta_{L}\right)$ and $V_{H}^{*}$ using Lemma B.2 and letting $g=1$, we can rewrite inequality (B17) as (**).

We are now ready to establish the following result in the high-polarization case.

LEMMA B.8: Suppose $\frac{\theta_{H}}{\theta_{L}}>\frac{1-\delta p}{\delta(1-p)}, \psi \geq \theta_{L}^{*}$ and condition $(* *)$ holds. Then, there exists an equilibrium strategy-payoff pair $\sigma=\left(\left(\pi^{H}, \alpha^{H}\right),\left(\pi^{L}, \alpha^{L}\right)\right)$ and $\left(V_{H}, W_{H}, V_{L}, W_{L}\right)$ that satisfies $\left(G 1^{\prime}\right)-\left(G 4^{\prime}\right)$.

The proof of Lemma B.8 is given in the next subsection.

The next two lemmas show that $\psi \geq \theta_{L}^{*}$ holds when polarization is not too high and condition $(* *)$ holds when parties' values of the public good are not too high.

LEMMA B.9: In the high-polarization case, $\psi \geq \theta_{L}^{*}$ holds when polarization is not too high.

Proof: Recall that $\frac{\theta_{H}}{\theta_{L}}>\frac{1-\delta p}{\delta(1-p)}$ in the high-polarization case. Since $\psi$ is continuous in $\theta_{H}$ and $\theta_{L}$, we only need to show that $\psi>\theta_{L}^{*}$ when $\frac{\theta_{H}}{\theta_{L}}=\frac{1-\delta p}{\delta(1-p)}$, which is equivalent to $\theta_{H}^{*}=\theta_{H}+\theta_{L}$. We substitute $\theta_{H}^{*}=\theta_{H}+\theta_{L}$ into (B10). Then (B10) simplifies to $\frac{\delta(1-p)(1-g)}{(1-\delta)(1+\delta-2 \delta p)}+\frac{\theta_{L}}{1-\delta} \ln (g)=\frac{\delta(1-p)\left(1-\left[\theta_{H}+\theta_{L}\right]\right)}{(1-\delta)(1+\delta-2 \delta p)}+\frac{\theta_{L}}{1-\delta} \ln \left(\theta_{H}+\theta_{L}\right)$. This implies $\psi=\theta_{H}+\theta_{L}$. So $\psi>\theta_{L}^{*}$ is equivalent to $\frac{\theta_{H}}{\theta_{L}}>\frac{\delta(1-p)}{1-\delta p}$, which always holds since $\frac{\theta_{H}}{\theta_{L}} \geq 1>\frac{\delta(1-p)}{1-\delta p}$.

LEMMA B.10: In the high-polarization case, (**) holds when parties' values of the public good are not too high.

Proof: To show that $(* *)$ holds when parties' values of the public good are not too high, we show that it holds strictly as the value of $\theta_{H}$ and $\theta_{L}$ approach zero. By l'Hôpital's rule, as $\theta_{H} \rightarrow 0$ and $\theta_{L} \rightarrow 0$, the left-hand side of condition $(* *)$ approaches 1 and the right-hand side of condition $(* *)$ approaches 0 . So $(* *)$ holds strictly, and by continuity, (**) also holds when $\theta_{H}$ and $\theta_{L}$ are not too high.

Proposition 4 follows from Lemmas B.3 and B.8-B.10. Specifically, for part $(i)$ of Proposition 4, notice first that $\left(\mathrm{G}^{\prime}\right)-\left(\mathrm{G} 4^{\prime}\right)$ imply proposal strategies for party $L$ that are the same as those given in Proposition 3. Hence party $L$ proposes higher levels of public good spending when it is mandatory than when it is discretionary. Similarly, proposal strategies for party $H$ given in $\left(\mathrm{G}^{\prime}\right)$ shows that the level of public good proposed by party $H$ is weakly higher than $\theta_{H}+\theta_{L}$, the efficient level, and therefore higher than what it proposes when public spending is discretionary. 
For Proposition $4(i i)$, observe again in (G3') that when $g \leq \underline{g}_{H}$, or $g \geq g_{H}^{*}$, party $H$ 's ideal $g_{H}^{*}$ is proposed. The value of $g_{H}^{*}$ given in Lemma B.3 is higher than the efficient level under high polarization.

Proposition 4 (iii) follows from inspecting the proposal strategies given in $\left(\mathrm{G} 1^{\prime}\right)-\left(\mathrm{G} 4^{\prime}\right)$.

B6. Proof of Lemma B.8 (High-polarization equilibrium characterization)

We proceed by first conjecturing an equilibrium strategy-payoff pair and then verifying that it satisfies guesses $\left(\mathrm{G}^{\prime}\right)-\left(\mathrm{G} 4^{\prime}\right)$, equilibrium conditions (E1)-(E3), and our assumption on $\alpha^{i}$ that all proposals made on the equilibrium path are accepted, when $\frac{\theta_{H}}{\theta_{L}}>\frac{1-\delta p}{\delta(1-p)}, \psi \geq \theta_{L}^{*}$, and condition $(* *)$ holds.

We conjecture an equilibrium strategy-payoff pair such that for any $i, j \in\{H, L\}$ with $j \neq i$, the acceptance strategy $\alpha^{i}(g, \mathbf{z})$ satisfies (E1), the proposal strategies are

$$
\begin{gathered}
\gamma^{L}(g)= \begin{cases}g_{L}^{*} & \text { for } g \leq g_{L}^{*}, \\
g & \text { for } g_{L}^{*} \leq g \leq \theta_{H}+\theta_{L}, \\
\theta_{H}+\theta_{L} & \text { for } \theta_{H}+\theta_{L} \leq g,\end{cases} \\
\chi_{H}^{L}(g)= \begin{cases}0 & \text { for } g \leq \theta_{H}+\theta_{L}, \\
K_{H}(g)-\theta_{H} \ln \left(\theta_{H}+\theta_{L}\right)-\delta\left[(1-p) V_{H}\left(\theta_{H}+\theta_{L}\right)+p W_{H}\left(\theta_{H}+\theta_{L}\right)\right] & \text { for } \theta_{H}+\theta_{L} \leq g,\end{cases} \\
\gamma^{H}(g)= \begin{cases}g_{H}^{*} & \text { for } g \leq \underline{g}_{H}, \\
g^{\prime} \in\left[\theta_{H}+\theta_{L}, g_{H}^{*}\right] & \text { for } \underline{g}_{H} \leq g \leq \tilde{g}_{H}, \\
\theta_{H}+\theta_{L} & \text { for } \tilde{g}_{H} \leq g \leq \theta_{H}+\theta_{L}, \\
g & \text { for } \theta_{H}+\theta_{L} \leq g \leq g_{H}^{*}, \\
g_{H}^{*} & \text { for } g_{H}^{*} \leq g,\end{cases}
\end{gathered}
$$$$
\chi_{L}^{H}(g)= \begin{cases}0 & \text { for } g \leq \tilde{g}_{H}, \\ K_{L}(g)-\theta_{L} \ln \left(\theta_{H}+\theta_{L}\right)-\delta\left[(1-p) V_{L}\left(\theta_{H}+\theta_{L}\right)+p W_{L}\left(\theta_{H}+\theta_{L}\right)\right] & \text { for } g \in\left[\tilde{g}_{H}, \theta_{H}+\theta_{L}\right] \\ 0 & \text { for } g \geq \theta_{H}+\theta_{L}\end{cases}
$$

and $\chi_{i}^{i}(g)=1-\gamma^{i}(g)-\chi_{j}^{i}(g)$, where $g_{L}^{*}=\theta_{L}, g_{H}^{*}=\theta_{H}^{*}, \underline{g}_{H}$ satisfies (B10), $\tilde{g}_{H}$ satisfies (B15), $g^{\prime}$ satisfies

$$
\theta_{L} \ln \left(g^{\prime}\right)+\delta\left[(1-p) V_{L}\left(g^{\prime}\right)+p W_{L}\left(g^{\prime}\right)\right]=K_{L}(g)
$$


and the associated payoff functions are

$$
\begin{gathered}
V_{L}(g)= \begin{cases}V_{L}^{*} & \text { for } g \leq g_{L}^{*}, \\
\frac{1}{1-\delta p}\left(1-g+\theta_{L} \ln (g)+\delta(1-p) W_{L}^{*}\right) & \text { for } g_{L}^{*} \leq g \leq \underline{g}_{H}, \\
\frac{(1-\delta p)(1-g)}{(1-\delta)(1+\delta-2 \delta p)}+\frac{\theta_{L}}{1-\delta} \ln (g) & \text { for } \underline{g}_{H} \leq g \leq \theta_{H}+\theta_{L}, \\
B_{L}^{1} g+C_{L}^{1} \ln (g)+D_{L}^{1} & \text { for } \theta_{H}+\theta_{L} \leq g \leq g_{H}^{*}, \\
C_{L}^{2} \ln (g)+D_{L}^{2} & \text { for } g_{H}^{*} \leq g,\end{cases} \\
W_{L}(g)= \begin{cases}W_{L}^{*} & \text { for } g \leq \underline{g}_{H} \text { and } g \geq g_{H}^{*}, \\
\frac{1}{1-\delta p}\left[\theta_{L} \ln (g)+\delta(1-p) V_{L}(g)\right] & \text { for } \underline{g}_{H} \leq g \leq g_{H}^{*},\end{cases} \\
V_{H}(g)= \begin{cases}V_{H}^{*} & \text { for } g \leq \underline{g}_{H}, \\
\frac{(1-\delta p)\left(1-\gamma^{H}(g)\right)}{(1-\delta)(1+\delta-2 \delta p)}+\frac{\theta_{H}}{1-\delta} \ln \left(\gamma^{H}(g)\right) & \text { for } \underline{g}_{H} \leq g \leq \tilde{g}_{H}, \\
B_{H}^{1} g+C_{H}^{1} \ln (g)+D_{H}^{1} & \text { for } \tilde{g}_{H} \leq g \leq \theta_{H}+\theta_{L}, \\
\frac{(1-\delta p)(1-g)}{(1-\delta)(1+\delta-2 \delta p)}+\frac{\theta_{H}}{1-\delta} \ln (g) & \text { for } \theta_{H}+\theta_{L} \leq g \leq g_{H}^{*}, \\
V_{H}^{*} & \text { for } g_{H}^{*} \leq g,\end{cases} \\
W_{H}(g)= \begin{cases}W_{H}^{*} \\
\frac{1}{1-\delta p}\left[\theta_{H} \ln (g)+\delta(1-p) V_{H}(g)\right] & \text { for } g_{L}^{*} \leq g,\end{cases}
\end{gathered}
$$

where $B_{i}^{1}=\frac{\delta(1-p)}{(1-\delta)(1+\delta-2 \delta p)}, C_{i}^{1}=-\frac{\theta_{j}}{1-\delta}, D_{i}^{1}=\frac{1-\delta p}{(1-\delta)(1+\delta-2 \delta p)}+\frac{\left(\theta_{H}+\theta_{L}\right)\left[\ln \left(\theta_{H}+\theta_{L}\right)-1\right]}{1-\delta}, C_{L}^{2}=$ $-\frac{\theta_{H}}{1-\delta p}, D_{L}^{2}=\frac{\delta(1-p)}{(1-\delta)(1-\delta p)}\left(\theta_{H}-\ln \left(g_{H}^{*}\right)\right)+D_{L}^{1}$, and

$$
\begin{aligned}
W_{L}^{*} & =\frac{\delta(1-p)}{(1+\delta-2 \delta p)(1-\delta)}\left(1-g_{H}^{*}\right)+\frac{\theta_{L}}{1-\delta} \ln \left(g_{H}^{*}\right), \\
V_{L}^{*} & =\frac{1}{1-\delta p}\left[1-\theta_{L}+\theta_{L} \ln \left(\theta_{L}\right)+\delta(1-p) W_{L}^{*}\right], \\
V_{H}^{*} & =\frac{(1-\delta p)\left(1-g_{H}^{*}\right)}{(1+\delta-2 \delta p)(1-\delta)}+\frac{\theta_{H}}{1-\delta} \ln \left(g_{H}^{*}\right), \\
W_{H}^{*} & =\frac{1}{1-\delta p}\left[\theta_{H} \ln \left(g_{L}^{*}\right)+\delta(1-p) V_{H}^{*}\right] .
\end{aligned}
$$

We next verify that this conjecture satisfies $\left(\mathrm{G} 1^{\prime}\right)-\left(\mathrm{G} 4^{\prime}\right)$.

For $\left(\mathrm{G} 1^{\prime}\right)$, since $g_{L}^{*}=\theta_{L}$ and $g_{H}^{*}=\theta_{H}^{*}$, clearly $g_{L}^{*}<\theta_{H}+\theta_{L}<g_{H}^{*}$ in the high-polarization case, and it only remains to show that $g_{i}^{*} \in \arg \max f_{i}(g)$. In Claim B.4 below, we show that (i) $g_{H}^{*} \in \arg \max f_{H}(g)$, and (ii) $g_{L}^{*} \in \arg \max f_{L}(g)$ when $\psi \geq \theta_{L}^{*}$, where $\psi$ is defined in (B10).

Since $V_{i}$ and $W_{i}$ are continuous under our conjecture of the equilibrium strategy-payoff pair, 
$f_{i}$ is continuous. It is also piecewise differentiable. Specifically,

$$
\begin{gathered}
f_{L}^{\prime}(g)= \begin{cases}-1+\frac{\theta_{L}}{g} & \text { for } g<g_{L}^{*}, \\
\frac{1}{1-\delta p}\left[-1+\frac{\theta_{L}}{g}\right] & \text { for } g \in\left(g_{L}^{*}, \underline{g}_{H}\right), \\
-\frac{1-\delta p}{(1-\delta)(1+\delta-2 \delta p)}+\frac{\theta_{L}}{(1-\delta) g} & \text { for } g \in\left(\underline{g}_{H}, \theta_{H}+\theta_{L}\right), \\
-1+\frac{1+\delta-2 \delta p}{1-\delta p} \frac{\theta_{L}}{g}+\frac{\delta(p+\delta-2 \delta p)}{1-\delta p}\left(B_{L}^{1}+\frac{C_{L}^{1}}{g}\right) & \text { for } g \in\left(\theta_{H}+\theta_{L}, g_{H}^{*}\right), \\
-1+\frac{1+\delta-2 \delta p}{1-\delta p} \frac{\theta_{L}}{g}+\frac{\delta(p+\delta-2 \delta p)}{1-\delta p} \frac{C_{L}^{2}}{g} & \text { for } g \geq g_{H}^{*},\end{cases} \\
f_{H}^{\prime}(g)= \begin{cases}-1+\frac{\theta_{H}}{g} & \text { for } g<g_{L}^{*}, \\
-1+\frac{1+\delta-2 \delta p}{1-\delta p} \frac{\theta_{H}}{g} & \text { for } g \in\left(g_{L}^{*}, g_{H}\right), \\
-1+\frac{1+\delta-2 \delta p}{1-\delta p} \frac{\theta_{H}}{g}+\frac{\delta(p+\delta-2 \delta p)}{1-\delta p}\left(-\frac{1-\delta p}{(1-\delta)(1+\delta-2 \delta p)}+\frac{\theta_{H}}{(1-\delta) \gamma^{H}(g)}\right) \frac{d \gamma^{H}(g)}{d g} & \text { for } g \in\left(\underline{g}_{H}, \tilde{g}_{H}\right), \\
-1+\frac{1+\delta-2 \delta p}{1-\delta p} \frac{\theta_{H}}{g}+\frac{\delta(p+\delta-2 \delta p)}{1-\delta p}\left(B_{H}^{1}+\frac{C_{H}^{1}}{g}\right) & \text { for } g \in\left(\tilde{g}_{H}, \theta_{H}+\theta_{L}\right), \\
-\frac{1-\delta p}{(1-\delta)(1+\delta-2 \delta p)}+\frac{\theta_{H}}{(1-\delta) g} & \text { for } g \in\left(\theta_{H}+\theta_{L}, g_{H}^{*}\right), \\
-1+\frac{1+\delta-2 \delta p}{1-\delta p} \frac{\theta_{H}}{g} & \text { for } g>g_{H}^{*} .\end{cases}
\end{gathered}
$$

CLAIM B.4: Under our conjecture of the equilibrium strategy-payoff pair, $(i) g_{H}^{*} \in \arg \max f_{H}(g)$, and (ii) if $\psi \geq \theta_{L}^{*}$, then $g_{L}^{*} \in \arg \max f_{L}(g)$.

Proof: Part (i): We show $f_{H}(g)$ is strictly increasing for $g \in\left(\tilde{g}_{H}, g_{H}^{*}\right)$, and strictly decreasing for $g>g_{H}^{*}$; hence $g_{H}^{*}=\arg \max _{g>\tilde{g}_{H}} f_{H}(g)$ by continuity of $f_{H}(g)$. Further, we show $f_{H}(g) \leq f_{H}\left(g_{H}^{*}\right)$ for $g \in\left(\underline{g}_{H}, \tilde{g}_{H}\right)$, and $f_{H}(g)$ is strictly increasing for $g<\underline{g}_{H}$. Hence, $g_{H}^{*} \in \arg \max f_{H}(g)$ by continuity of $f_{H}(g)$.

- $g<g_{L}^{*}: f_{H}^{\prime}(g)$ is decreasing. At $g_{L}^{*}=\theta_{L}, f_{H}^{\prime}\left(g_{L}^{*}\right)>0$, hence for $g<g_{L}^{*}, f_{H}^{\prime}(g)>0$.

- $g \in\left(g_{L}^{*}, \underline{g}_{H}\right): f_{H}^{\prime}(g)$ is decreasing. Since $\underline{g}_{H}<g_{H}^{*}$ and $f_{H}^{\prime}(g)=-1+\frac{g_{H}^{*}}{g}$, it follows that $f_{H}^{\prime}(g)>0$ for $g \in\left(g_{L}^{*}, \underline{g}_{H}\right)$.

- $g \in\left(\underline{g}_{H}, \tilde{g}\right)$ : We compare $f_{H}(g)$ in this range to $f_{H}\left(g_{H}^{*}\right)$. First define the functions

$$
\begin{aligned}
n(x) & =1-x+\frac{\theta_{H}(1+\delta-2 \delta p)}{1-\delta p} \ln (x), \text { and } \\
m(y) & =\frac{\delta(p+\delta-2 \delta p)}{1-\delta p}\left[\frac{(1-\delta p)(1-y)}{(1-\delta)(1+\delta-2 \delta p)}+\frac{\theta_{H}}{1-\delta} \ln (y)\right] .
\end{aligned}
$$

By these definitions $f_{H}\left(g_{H}^{*}\right)=n\left(g_{H}^{*}\right)+m\left(g_{H}^{*}\right)$, and $f_{H}(g)=n(g)+m\left(\gamma^{H}(g)\right)$ for $g \in\left(\underline{g}_{H}, \tilde{g}_{H}\right)$. Further note that $g_{H}^{*}=\arg \max n(x)$, and $g_{H}^{*}=\arg \max m(y)$, hence $n\left(g_{H}^{*}\right) \geq n(g)$ and $m\left(g_{H}^{*}\right) \geq m\left(\gamma^{H}(g)\right)$ for all $g$, so $f_{H}\left(g_{H}^{*}\right)>f_{H}(g)$ for $g \in\left(\underline{g}_{H}, \tilde{g}\right)$.

- $g \in\left(\tilde{g}_{H}, \theta_{H}+\theta_{L}\right): f_{H}^{\prime}(g)$ strictly decreasing. Since $f_{H}^{\prime}\left(\theta_{H}+\theta_{L}\right)=\frac{\theta_{H} \delta(1-p)-\theta_{L}(1-\delta p)}{(1-\delta)(1+\delta-2 \delta p)\left(\theta_{H}+\theta_{L}\right)}>$ $0, f_{H}^{\prime}(g)>0$ everywhere in this interval. 
- $g \in\left(\theta_{H}+\theta_{L}, g_{H}^{*}\right): f_{H}^{\prime}(g)$ strictly decreasing. Since $-\frac{1-\delta p}{(1-\delta)(1+\delta-2 \delta p)}+\frac{\theta_{H}}{(1-\delta) g_{H}^{*}}=0$, it follows that for $g \in\left(\theta_{H}+\theta_{L}, g_{H}^{*}\right), f_{H}^{\prime}(g)>0$.

- $g>g_{H}^{*}: f_{H}^{\prime}(g)=-1+\frac{g_{H}^{*}}{g}<0$.

Part (ii): We show $f_{L}(g)$ is strictly increasing for $g<g_{L}^{*}$ and strictly decreasing for $g>g_{L}^{*}$ and therefore $g_{L}^{*} \in \arg \max f_{L}(g)$.

- $g<g_{L}^{*}: f_{L}^{\prime}(g)>0$.

- $g \in\left(g_{L}^{*}, \underline{g}_{H}\right): f_{L}^{\prime}(g)$ is strictly decreasing. Since $f_{L}^{\prime}(g)=\frac{1}{1-\delta p}\left[-1+\frac{\theta_{L}}{g}\right]$, it follows that $f_{L}^{\prime}(g)<0$ for $g \in\left(g_{L}^{*}, \underline{g}_{H}\right)$.

- $g \in\left(\underline{g}_{H}, \theta_{H}+\theta_{L}\right): f_{L}^{\prime}(g)$ is strictly decreasing. Since $\underline{g}_{H}=\psi$ by Lemma B.5, we have $\underline{g}_{H}=\psi \geq \theta_{L}^{*}$. Since $-\frac{1-\delta p}{(1-\delta)(1+\delta-2 \delta p)}+\frac{\theta_{L}}{(1-\delta) g}=0$ if $g=\frac{\theta_{L}(1+\delta-2 \delta p)}{1-\delta p}$, it follows that $f_{L}^{\prime}(g)<0$ for all $g \in\left(\underline{g}_{H}, \theta_{H}+\theta_{L}\right)$.

- $g \in\left(\theta_{H}+\theta_{L}, g_{H}^{*}\right)$ : The monotonicity of $f_{L}^{\prime}(g)$ is determined by $\frac{(1+\delta-2 \delta p) \theta_{L}}{1-\delta p}+\frac{\delta(p+\delta-2 \delta p) C_{L}^{1}}{1-\delta p}$. If $\frac{(1+\delta-2 \delta p) \theta_{L}}{1-\delta p}+\frac{\delta(p+\delta-2 \delta p) C_{L}^{1}}{1-\delta p}>0$, then $f_{L}^{\prime}(g)$ is strictly decreasing in $g$. Since $-1+$ $\frac{1+\delta-2 \delta p}{1-\delta p} \frac{\theta_{L}}{g}+\frac{\delta(p+\delta-2 \delta p)}{1-\delta p}\left(B_{L}^{1}+\frac{C_{L}^{1}}{g}\right)=\frac{\theta_{L} \delta(1-p)-\theta_{H}(1-\delta p)}{(1-\delta)(1+\delta-2 \delta p)\left(\theta_{H}+\theta_{L}\right)} \leq 0$ if $g=\theta_{H}+\theta_{L}$, it follows that $f_{L}^{\prime}(g)<0$ for $g \in\left(\theta_{H}+\theta_{L}, g_{H}^{*}\right)$. If $\frac{(1+\delta-2 \delta p) \theta_{L}}{1-\delta p}+\frac{\delta(p+\delta-2 \delta p) C_{L}^{1}}{1-\delta p} \leq 0$, then $f_{L}^{\prime}(g)$ is weakly increasing in $g$. Since $-1+\frac{1+\delta-2 \delta p}{1-\delta p} \frac{\theta_{L}}{g}+\frac{\delta(p+\delta-2 \delta p)}{1-\delta p}\left(B_{L}^{1}+\frac{C_{L}^{1}}{g}\right)=-1+\frac{\theta_{L}}{\theta_{H}}-$ $\frac{\delta(p+\delta-2 \delta p)}{(1+\delta-2 \delta p)(1-\delta p)}<0$ when $g=g_{H}^{*}$, it follows that $f_{L}^{\prime}(g)<0$ for $g \in\left(\theta_{H}+\theta_{L}, g_{H}^{*}\right)$.

- $g>g_{H}^{*}$ : The monotonicity of $f_{L}^{\prime}(g)$ is determined by $\frac{(1+\delta-2 \delta p) \theta_{L}}{1-\delta p}+\frac{\delta(p+\delta-2 \delta p) C_{L}^{2}}{1-\delta p}$. If $\frac{(1+\delta-2 \delta p) \theta_{L}}{1-\delta p}+\frac{\delta(p+\delta-2 \delta p) C_{L}^{2}}{1-\delta p}>0$, then $f_{L}^{\prime}(g)$ is strictly decreasing in $g$. Since $-1+$ $\frac{1+\delta-2 \delta p}{1-\delta p} \frac{\theta_{L}}{g}+\frac{\delta(p+\delta-2 \delta p)}{1-\delta p} \frac{C_{L}^{2}}{g}=-1+\frac{\theta_{L}}{\theta_{H}}-\frac{\delta(p+\delta-2 \delta p)}{(1-\delta p)(1+\delta-2 \delta p)}<0$ if $g=g_{H}^{*}$, it follows that $f_{L}^{\prime}(g)<0$ for $g>g_{H}^{*}$. If $\frac{(1+\delta-2 \delta p) \theta_{L}}{1-\delta p}+\frac{\delta(p+\delta-2 \delta p) C_{L}^{2}}{1-\delta p} \leq 0$, then $f_{L}^{\prime}(g)$ is weakly increasing in $g$. In this case, $f_{L}^{\prime}(g)=-1+\frac{(1+\delta-2 \delta p) \theta_{L}}{1-\delta p}+\frac{\delta(p+\delta-2 \delta p) C_{L}^{2}}{1-\delta p}<0$ when $g=1$ and therefore $f_{L}^{\prime}(g)<0$ for $g>g_{H}^{*}$.

The conjectured equilibrium strategy-payoff pair clearly satisfies $\left(\mathrm{G}^{\prime}\right)-\left(\mathrm{G} 4^{\prime}\right)$ with the exception of $g_{L}^{*} \leq \underline{g}_{H}<\tilde{g}_{H}<\theta_{H}+\theta_{L}$. When $\psi \geq \theta_{L}^{*}$, we have $g_{L}^{*}=\theta_{L}<\theta_{L}^{*} \leq \psi=\underline{g}_{H}$. To verify that $\underline{g}_{H}<\tilde{g}_{H}<\theta_{H}+\theta_{L}$, we next establish some monotonicity properties of $\underline{K}_{L}$.

CLAIM B.5: Under our conjecture of the equilibrium strategy-payoff pair, $K_{L}(g)$ is strictly increasing for $g \in\left[0, \frac{\theta_{L}(1+\delta-2 \delta p)}{\delta(1-p)}\right)$ and strictly decreasing for $g \in\left(\frac{\theta_{L}(1+\delta-2 \delta p)}{\delta(1-p)}, 1\right]$.

Proof: Consider the following cases: 
- $g \leq g_{L}^{*}: K_{L}(g)=\theta_{L} \ln (g)+\delta\left[(1-p) V_{L}^{*}+p W_{L}^{*}\right]$, which is increasing in $g$.

- $g \in\left[g_{L}^{*}, \underline{g}_{H}\right]:$ In this case,

$$
K_{L}(g)=\theta_{L} \ln (g)+\frac{\delta(1-p)}{1-\delta p}\left(1-g+\theta_{L} \ln (g)+\delta(1-p) W_{L}^{*}\right)+\delta p W_{L}^{*} .
$$

Taking the derivative, we get

$$
K_{L}^{\prime}(g)=\frac{1+\delta-2 \delta p}{1-\delta p} \frac{\theta_{L}}{g}-\frac{\delta(1-p)}{1-\delta p},
$$

and $K_{L}^{\prime}(g)>0$ if and only if $g<\frac{1+\delta-2 \delta p}{\delta(1-p)} \theta_{L}$.

- $g \in\left[\underline{g}_{H}, \theta_{H}+\theta_{L}\right]:$ In this case,

$$
K_{L}(g)=\frac{\theta_{L}}{1-\delta p} \ln (g)+\frac{\delta(1-p)}{1-\delta p} V_{L}(g)=\frac{\theta_{L}}{1-\delta p} \ln (g)+\frac{\delta(1-p)}{1-\delta p}\left[\frac{(1-\delta p)(1-g)}{(1-\delta)(1+\delta-2 \delta p)}+\frac{\theta_{L}}{1-\delta} \ln (g)\right] .
$$

Taking the derivative, we get

$$
K_{L}^{\prime}(g)=\frac{1}{1-\delta}\left[\frac{-\delta(1-p)}{1+\delta-2 \delta p}+\frac{\theta_{L}}{g}\right]
$$

and $K_{L}^{\prime}(g)>0$ if and only if $g<\frac{1+\delta-2 \delta p}{\delta(1-p)} \theta_{L}$. Note that since $\theta_{H}+\theta_{L}>\frac{1+\delta-2 \delta p}{\delta(1-p)} \theta_{L}$ in the high-polarization case, $K_{L}^{\prime}(g)<0$ for $g \in\left(\frac{1+\delta-2 \delta p}{\delta(1-p)} \theta_{L}, \theta_{H}+\theta_{L}\right)$.

- $g \in\left[\theta_{H}+\theta_{L}, g_{H}^{*}\right]:$ In this case,

$$
K_{L}(g)=\frac{\theta_{L}}{1-\delta p} \ln (g)+\frac{\delta(1-p)}{1-\delta p} V_{L}(g)=\frac{\theta_{L}}{1-\delta p} \ln (g)+\frac{\delta(1-p)}{1-\delta p}\left(B_{L}^{1} g+C_{L}^{1} \ln (g)+D_{L}^{1}\right) .
$$

Taking the derivative, we get

$$
K_{L}^{\prime}(g)=\frac{1}{(1-\delta p)(1-\delta)}\left[\frac{(1-\delta) \theta_{L}-\delta(1-p) \theta_{H}}{g}+\frac{\delta^{2}(1-p)^{2}}{1+\delta-2 \delta p}\right],
$$

which is increasing in $g$ since $(1-\delta) \theta_{L}-\delta(1-p) \theta_{H}<0$ in the high-polarization case. Straightforward calculation shows that $K_{L}^{\prime}(g)<0$ for $g=g_{H}^{*}$. Hence, $K_{L}(g)$ is strictly decreasing for $g \in\left[\theta_{H}+\theta_{L}, g_{H}^{*}\right]$.

- $g \geq g_{H}^{*}$ : In this case,

$$
K_{L}(g)=\theta_{L} \ln (g)+\delta\left[(1-p) V_{L}(g)+p W_{L}(g)\right]=\theta_{L} \ln (g)+\delta(1-p)\left(C_{L}^{2} \ln (g)+D_{L}^{2}\right)+\delta p W_{L}^{*}
$$

Taking the derivative and substituting for $C_{L}^{2}$, we get

$$
K_{L}^{\prime}(g)=\frac{\theta_{L}}{g}-\frac{\delta(1-p) \theta_{H}}{(1-\delta p) g},
$$


which is negative since $\frac{\theta_{H}}{\theta_{L}}>\frac{1-\delta p}{\delta(1-p)}$ in the high-polarization case. Hence, $K_{L}(g)$ is strictly increasing for $g \in\left[0, \frac{\theta_{L}(1+\delta-2 \delta p)}{\delta(1-p)}\right)$ and strictly decreasing for $g \in\left(\frac{\theta_{L}(1+\delta-2 \delta p)}{\delta(1-p)}, 1\right]$.

Recall that in our conjectured equilibrium, $\underline{g}_{H}$ satisfies $K_{L}\left(\underline{g}_{H}\right)=K_{L}\left(g_{H}^{*}\right)$ and $\tilde{g}_{H}$ satisfies $K_{L}\left(\tilde{g}_{H}\right)=K_{L}\left(\theta_{H}+\theta_{L}\right)$. Since $K_{L}$ is continuous, $K_{L}(g)=-\infty$ when $g=0$, and $\frac{\theta_{L}(1+\delta-2 \delta p)}{\delta(1-p)}<$ $\theta_{H}+\theta_{L}<g_{H}^{*}$ in the high-polarization case, we have the following corollary of Claim B.5.

COROLLARY B.2: There exist $\underline{g}_{H}$ and $\tilde{g}_{H}$ where $\underline{g}_{H}<\tilde{g}_{H}<\frac{\theta_{L}(1+\delta-2 \delta p)}{\delta(1-p)}<\theta_{H}+\theta_{L}<g_{H}^{*}$ such that $K_{L}\left(\underline{g}_{H}\right)=K_{L}\left(g_{H}^{*}\right)$ and $K_{L}\left(\tilde{g}_{H}\right)=K_{L}\left(\theta_{H}+\theta_{L}\right)$.

We next verify that equilibrium conditions (E1)-(E3) are satisfied. Condition (E1) is satisfied by construction. The values $V_{L}^{*}, W_{L}^{*}, V_{H}^{*}$ and $W_{H}^{*}$ satisfy

$$
\begin{gathered}
V_{L}^{*}=1-g_{L}^{*}+\theta_{L} \ln \left(g_{L}^{*}\right)+\delta\left[p V_{L}^{*}+(1-p) W_{L}^{*}\right], \\
W_{L}^{*}=\theta_{L} \ln \left(g_{H}^{*}\right)+\delta\left[(1-p) V_{L}^{*}+p W_{L}^{*}\right], \\
V_{H}^{*}=1-g_{H}^{*}+\theta_{H} \ln \left(g_{H}^{*}\right)+\delta\left[p V_{H}^{*}+(1-p) W_{H}\left(g_{H}^{*}\right)\right], \\
W_{H}^{*}=\theta_{H} \ln \left(g_{L}^{*}\right)+\delta\left[(1-p) V_{H}^{*}+p W_{H}^{*}\right] .
\end{gathered}
$$

Together with Lemmas B.1, B.2 and B.4, these show that (E3) is satisfied, that is, these payoff functions are consistent with the strategy profile.

Recall $U_{i}^{P}(\mathbf{z})\left(U_{i}^{R}(\mathbf{z})\right)$ denotes party $i$ 's dynamic payoff when the implemented budget is $\mathbf{z}$ in the current period and party $i$ is the proposer (responder). The next claim establishes that all equilibrium proposals are accepted.

CLAIM B.6: Under our conjecture of the equilibrium strategy-payoff pair, if condition (**) holds, then $\alpha^{j}\left(g, \pi^{i}(g)\right)=1$ for all $g$ and all $i, j \in\{H, L\}, j \neq i$.

Proof: We omit the proof for $j=H$ since it is similar to that for Claim B.3. We use condition $(* *)$ in place of condition $(*)$.

Now consider $j=L$. If $g \leq g_{L}^{*}$, then $U_{L}^{R}\left(\pi^{H}(g)\right)=\theta_{L} \ln \left(g_{H}^{*}\right)+\delta\left[(1-p) V_{L}^{*}+p W_{L}^{*}\right] \geq$ $K_{L}(g)=\theta_{L} \ln \left(g_{H}\right)+\delta\left[(1-p) V_{L}^{*}+p W_{L}^{*}\right]$ and therefore $\alpha^{L}\left(g, \pi^{H}(g)\right)=1$.

If $g \in\left[g_{L}^{*}, \underline{g}_{H}\right]$, then $U_{L}^{R}\left(\pi^{H}(g)\right)=\theta_{L} \ln \left(g_{H}^{*}\right)+\delta\left[(1-p) V_{L}^{*}+p W_{L}^{*}\right]=K_{L}\left(g_{H}^{*}\right)$. Since $K_{L}\left(\underline{g}_{H}\right)=K_{L}\left(g_{H}^{*}\right)$ and $K_{L}(g)$ is increasing on $\left[g_{L}^{*}, \underline{g}_{H}\right]$ by Claim B.5, it follows that $U_{L}^{R}\left(\pi^{H}(g)\right) \geq$ $K_{L}(g)$ and therefore $\alpha^{L}\left(g, \pi^{H}(g)\right)=1$ for $g \in\left[g_{L}^{*}, \underline{g}_{H}\right]$.

If $g \in\left[\underline{g}_{H}, g_{H}^{*}\right]$, then $U_{L}^{R}\left(\pi^{H}(g)\right)=K_{L}(g)$ and $\alpha^{L}\left(g, \pi^{H}(g)\right)=1$.

If $g \in\left[g_{H}^{*}, 1\right]$, then $U_{L}^{R}\left(\pi^{H}(g)\right)=\theta_{L} \ln \left(g_{H}^{*}\right)+\delta\left[(1-p) V_{L}^{*}+p W_{L}^{*}\right]=K_{L}\left(g_{H}^{*}\right)$. Since $K_{L}(g)$ is decreasing on $\left[g_{H}^{*}, 1\right]$ by Claim B.5 and Corollary B.2, it follows that $U_{L}^{R}\left(\pi^{H}(g)\right) \geq K_{L}(g)$ and therefore $\alpha^{L}\left(g, \pi^{H}(g)\right)=1$ for $g \in\left[g_{H}^{*}, 1\right]$.

The remainder of the proof shows that (E2) is satisfied. The next claim establishes that $K_{H}(g)$ is increasing, which is useful later in the proof. 
CLAIM B.7: Under our conjecture of the equilibrium strategy-payoff pair, if $\psi \geq \theta_{L}^{*}$, then $K_{H}(g)$ is strictly increasing.

Proof: Consider the following cases.

- $g \leq g_{L}^{*}: K_{H}(g)=\theta_{H} \ln (g)+\delta\left[(1-p) V_{H}^{*}+p W_{H}^{*}\right]$, which is strictly increasing.

- $g \in\left[g_{L}^{*}, \underline{g}_{H}\right]: K_{H}(g)=\theta_{H} \ln (g)+\delta(1-p) V_{H}^{*}+\frac{\delta p}{1-\delta p}\left[\theta_{H} \ln (g)+\delta(1-p) V_{H}^{*}\right]$, which is strictly increasing.

- $g \in\left[\underline{g}_{H}, \tilde{g}_{H}\right]: K_{H}(g)=\frac{\theta_{H}}{1-\delta p} \ln (g)+\frac{\delta(1-p)}{1-\delta p} V_{H}(g)$, and $K_{H}^{\prime}(g)=\frac{\theta_{H}}{(1-\delta p) g}+\frac{\delta(1-p)}{1-\delta p} V_{H}^{\prime}(g)$. The function $V_{H}(g)$ is

$$
V_{H}(g)=\frac{(1-\delta p)\left(1-\gamma^{H}(g)\right)}{(1-\delta)(1+\delta-2 \delta p)}+\frac{\theta_{H}}{1-\delta} \ln \left(\gamma^{H}(g)\right),
$$

and $\gamma^{H}(g)$ is given by (B18), which implies

$$
\begin{aligned}
& \frac{\theta_{L}}{1-\delta p} \ln \left(\gamma^{H}(g)\right)+\frac{\delta(1-p)}{1-\delta p}\left[\frac{\delta(1-p)}{(1-\delta)(1+\delta-2 \delta p)} \gamma^{H}(g)-\frac{\theta_{H}}{1-\delta} \ln \left(\gamma^{H}(g)\right)+D_{L}^{1}\right] \\
= & \frac{\theta_{L}}{1-\delta p} \ln (g)+\frac{\delta(1-p)}{1-\delta p}\left[\frac{(1-\delta p)(1-g)}{(1-\delta)(1+\delta-2 \delta p)}+\frac{\theta_{L}}{1-\delta} \ln (g)\right] .
\end{aligned}
$$

Rearranging (B23) gives

$$
\ln \left(\gamma^{H}(g)\right)=\frac{1-\delta p}{\theta_{L}(1-\delta)-\theta_{H} \delta(1-p)}\left[\theta_{L} \ln (g)+\frac{\delta(1-p)(1-g)}{1+\delta-2 \delta p}-\frac{\delta^{2}(1-p)^{2} \gamma^{H}(g)}{(1-\delta p)(1+\delta-2 \delta p)}-\frac{\delta(1-p)(1-\delta)}{1-\delta p} D_{L}^{1}\right] .
$$

Substituting $\ln \left(\gamma^{H}(g)\right)$ into $V_{H}(g)$ and taking the derivative, we have

$$
\begin{aligned}
V_{H}^{\prime}(g)= & \frac{\theta_{H} \theta_{L}(1-\delta p)}{(1-\delta)\left[\theta_{L}(1-\delta)-\theta_{H} \delta(1-p)\right] g}-\frac{\theta_{H} \delta(1-p)(1-\delta p)}{(1-\delta)\left[\theta_{L}(1-\delta)-\theta_{H} \delta(1-p)\right](1+\delta-2 \delta p)} \\
& -\frac{d \gamma^{H}(g)}{d g} \frac{\theta_{L}(1-\delta p)-\theta_{H} \delta(1-p)}{(1+\delta-2 \delta p)\left[\theta_{L}(1-\delta)-\theta_{H} \delta(1-p)\right]},
\end{aligned}
$$

and $K_{H}^{\prime}(g)=A(g)+B(g)$ where

$$
\begin{aligned}
& A(g)=\frac{\theta_{H}}{(1-\delta p) g}+\frac{\theta_{H} \delta(1-p)}{(1-\delta)\left[\theta_{L}(1-\delta)-\theta_{H} \delta(1-p)\right]}\left[\frac{\theta_{L}}{g}-\frac{\delta(1-p)}{1+\delta-2 \delta p}\right], \\
& B(g)=-\frac{\delta(1-p)\left[\theta_{L}(1-\delta p)-\theta_{H} \delta(1-p)\right]}{(1-\delta p)(1+\delta-2 \delta p)\left[\theta_{L}(1-\delta)-\theta_{H} \delta(1-p)\right]} \frac{d \gamma^{H}(g)}{d g} .
\end{aligned}
$$

We first show $A(g)>0$. Suppose the coefficient on $\frac{1}{g}$ is positive. Then $A(g)$ is strictly decreasing and is minimized at $g=\tilde{g}_{H}$. By Corollary B.2, $\tilde{g}_{H}<\frac{\theta_{L}(1+\delta-2 \delta p)}{\delta(1-p)}$. Since $A(g)=\frac{\theta_{H} \delta(1-p)}{\theta_{L}(1-\delta p)(1+\delta-2 \delta p)}>0$ when $g=\frac{\theta_{L}(1+\delta-2 \delta p)}{\delta(1-p)}$, it follows that $A(g)>0$ for $g \in$ $\left[\underline{g}_{H}, \tilde{g}_{H}\right]$ in this case. Now suppose the coefficient on $\frac{1}{g}$ is negative, then $A(g)$ is strictly increasing and is minimized at $g=\underline{g}_{H}$. We have $\underline{g}_{H}=\psi \geq \theta_{L}^{*}$. When $g=\theta_{L}^{*}$, $A(g)=\frac{\theta_{H}\left[\theta_{H} \delta(1-p)-\theta_{L}(1-\delta p)\right.}{\theta_{L}(1+\delta-2 \delta p)\left[\theta_{H} \delta(1-p)-\theta_{L}(1-\delta)\right]}$, which is strictly positive in the high-polarization 
case. Finally suppose the coefficient on $\frac{1}{g}$ is zero, then $A(g)>0$. It follows that $A(g)>0$ for $g \in\left[\underline{g}_{H}, \tilde{g}_{H}\right]$.

We next show that $B(g)>0$. Since $\gamma^{H}(g)$ satisfies (B23), by the implicit function theorem,

$$
\frac{d \gamma^{H}(g)}{d g}=\frac{\gamma^{H}(g)(1-\delta p)\left[\theta_{L}(1+\delta-2 \delta p)-g \delta(1-p)\right]}{g\left[(1+\delta-2 \delta p)\left(\theta_{L}(1-\delta)-\theta_{H} \delta(1-p)\right)+\gamma^{H}(g) \delta^{2}(1-p)^{2}\right]} .
$$

At $\gamma^{H}(g)=g_{H}^{*}$ the denominator of $\frac{d \gamma^{H}(g)}{d g}$ is negative. Since the denominator is increasing in $\gamma^{H}(g)$ and $\gamma^{H}(g) \leq g_{H}^{*}$, the denominator is negative. Since $g \leq \tilde{g}_{H}<\frac{\theta_{L}(1+\delta-2 \delta p)}{\delta(1-p)}$, the numerator is positive, and therefore $\frac{d \gamma^{H}(g)}{d g}<0$. Since this is the high-polarization case and $\frac{d \gamma^{H}(g)}{d g}<0$, it follows that $B(g)>0$.

To summarize, $K_{H}^{\prime}(g)=A(g)+B(g)>0$ for $g \in\left[\underline{g}_{H}, \tilde{g}_{H}\right]$.

- $g \in\left[\tilde{g}_{H}, \theta_{H}+\theta_{L}\right]: K_{H}(g)=\frac{\theta_{H}}{1-\delta p} \ln (g)+\frac{\delta(1-p)}{1-\delta p} V_{H}(g)$. Substituting for $V_{H}(g)$ and taking the derivative, we get

$$
K_{H}^{\prime}(g)=\frac{(1-\delta) \theta_{H}-\delta(1-p) \theta_{L}}{(1-\delta p)(1-\delta) g}+\frac{\delta(1-p)}{1-\delta p} B_{H}^{1}
$$

If $(1-\delta) \theta_{H}-\delta(1-p) \theta_{L}>0$, then, $K_{H}^{\prime}(g)>0$ since $B_{H}^{1}>0$.

If $(1-\delta) \theta_{H}-\delta(1-p) \theta_{L}<0$, then $K_{H}^{\prime}(g)$ is increasing in $g$. We have $\tilde{g}_{H}>\underline{g}_{H}=\psi \geq \theta_{L}^{*}$. Plugging $g=\theta_{L}^{*}$ in $(\mathrm{B} 25)$, we get $K_{H}^{\prime}(g)=\frac{\theta_{H}(1-\delta p)-\theta_{L} \delta(1-p)}{(1-\delta p)(1+\delta-2 \delta p) \theta_{L}}>0$, and therefore $K_{H}(g)$ is strictly increasing for $g \in\left[\tilde{g}_{H}, \theta_{H}+\theta_{L}\right]$.

- $g \in\left[\theta_{H}+\theta_{L}, g_{H}^{*}\right]: K_{H}(g)=\frac{\theta_{H}}{1-\delta p} \ln (g)+\frac{\delta(1-p)}{1-\delta p} V_{H}(g)$. Substituting for $V_{H}(g)$ and taking the derivative, we get

$$
K_{H}^{\prime}(g)=\frac{\theta_{H}}{(1-\delta) g}-\frac{\delta(1-p)}{(1-\delta)(1+\delta-2 \delta p)},
$$

which is strictly higher than 0 for $g \leq g_{H}^{*}$.

- $g>g_{H}^{*}: K_{H}(g)=\frac{\theta_{H}}{1-\delta p} \ln (g)+\frac{\delta(1-p)}{1-\delta p} V_{H}^{*}$, which is strictly increasing.

We next show that the proposer has no profitable one-shot deviation. We omit the proof for party $L$ since it is similar to that in the proof of Lemma 7 .

We next establish monotonicity properties of $U_{H}^{P}(\mathbf{z})$, which is useful for later in the proof. For any status quo $g$, consider proposals $\mathbf{z}^{\prime}=\left(g^{\prime}, x_{H}^{\prime}, x_{L}^{\prime}\right)$ such that the responder's acceptance constraint (5) is binding. That is,

$$
x_{L}^{\prime}=K_{L}(g)-\theta_{L} \ln \left(g^{\prime}\right)-\delta\left[(1-p) V_{L}\left(g^{\prime}\right)+p W_{L}\left(g^{\prime}\right)\right]=K_{L}(g)-K_{L}\left(g^{\prime}\right) .
$$


Substituting in the proposer's payoff function, we get $U_{H}^{P}\left(\mathbf{z}^{\prime}\right)=1-g^{\prime}-x_{L}^{\prime}+\theta_{H} \ln \left(g^{\prime}\right)+$ $\delta\left[p V_{H}\left(g^{\prime}\right)+(1-p) W_{H}\left(g^{\prime}\right)\right]$, which implies

$$
\frac{\partial U_{H}^{P}}{\partial g^{\prime}}=-1+\frac{\theta_{H}+\theta_{L}}{g^{\prime}}+\delta\left[(1-p) V_{L}^{\prime}\left(g^{\prime}\right)+p W_{L}^{\prime}\left(g^{\prime}\right)\right]+\delta\left[p V_{H}^{\prime}\left(g^{\prime}\right)+(1-p) W_{H}^{\prime}\left(g^{\prime}\right)\right] .
$$

Substituting for $V_{L}^{\prime}, W_{L}^{\prime}, V_{H}^{\prime}, W_{H}^{\prime}$, we get a closed-form solution for $\frac{\partial U_{H}^{P}}{\partial g^{\prime}}$ except when $g \in$ $\left(\underline{g}_{H}, \tilde{g}_{H}\right)$. Specifically, if $g^{\prime}<g_{L}^{*}$, then $\frac{\partial U_{H}^{P}}{\partial g^{\prime}}=\frac{\theta_{H}+\theta_{L}}{g^{\prime}}-1>0$; if $g^{\prime} \in\left(g_{L}^{*}, \underline{g}_{H}\right)$, then $\frac{\partial U_{H}^{P}}{\partial g^{\prime}}=$ $\frac{1+\delta-2 \delta p}{1-\delta p}\left(\frac{\theta_{H}+\theta_{L}}{g^{\prime}}-1\right)>0$; if $g^{\prime} \in\left(\tilde{g}_{H}, \theta_{H}+\theta_{L}\right)$, then $\frac{\partial U_{H}^{P}}{\partial g^{\prime}}=\frac{1+\delta-2 \delta p}{1-\delta p}\left(\frac{\theta_{H}+\theta_{L}}{g^{\prime}}-1\right)>0$; if $g^{\prime} \in\left(\theta_{H}+\theta_{L}, g_{H}^{*}\right)$, then $\frac{\partial U_{H}^{P}}{\partial g^{\prime}}=\frac{1}{1-\delta p}\left(\frac{\theta_{H}+\theta_{L}}{g^{\prime}}-1\right)<0$; if $g^{\prime}>g_{H}^{*}$, then $\frac{\partial U_{H}^{P}}{\partial g^{\prime}}=\frac{\theta_{H}+\theta_{L}}{g^{\prime}}-1<0$.

Note that $\frac{\partial U_{H}^{P}}{\partial g^{\prime}}=f_{H}^{\prime}\left(g^{\prime}\right)+K_{L}^{\prime}\left(g^{\prime}\right)$. Also, if $g^{\prime} \in\left(\underline{g}_{H}, \tilde{g}_{H}\right)$, then $\frac{d \gamma^{H}\left(g^{\prime}\right)}{d g^{\prime}}=\frac{K_{L}^{\prime}\left(g^{\prime}\right)}{K_{L}^{\prime}\left(\gamma^{H}\left(g^{\prime}\right)\right)}$. Hence, for $g^{\prime} \in\left(\underline{g}_{H}, \tilde{g}_{H}\right)$,

$$
\frac{\partial U_{H}^{P}}{\partial g^{\prime}}=-1+\frac{1+\delta-2 \delta p}{1-\delta p} \frac{\theta_{H}}{g^{\prime}}+K_{L}^{\prime}\left(g^{\prime}\right) C\left(g^{\prime}\right)
$$

where

$$
C\left(g^{\prime}\right)=1+\frac{\delta(p+\delta-2 \delta p)\left[-(1-\delta p) \gamma^{H}\left(g^{\prime}\right)+(1+\delta-2 \delta p) \theta_{H}\right]}{\left[(1-\delta) \theta_{L}-\delta(1-p) \theta_{H}\right](1+\delta-2 \delta p)+\gamma^{H}\left(g^{\prime}\right) \delta^{2}(1-p)^{2}} .
$$

We verify that $C\left(g^{\prime}\right)>0$ in the high-polarization case where $\frac{\theta_{H}}{\theta_{L}}>\frac{1-\delta p}{\delta(1-p)}$. Since $K_{L}^{\prime}\left(g^{\prime}\right)>0$ for $g^{\prime}<\tilde{g}_{H}$ by Claim B.5 and Corollary B.2, it follows that $\frac{\partial U_{H}^{P}}{\partial g^{\prime}}>0$ for $g^{\prime} \in\left(\underline{g}_{H}, \tilde{g}_{H}\right)$.

Below we show that proposer $H$ has no profitable one-shot deviation.

- $g \leq \underline{g}_{H}$ or $g \geq g_{H}^{*}$ : In this case, $\gamma^{H}(g)=g_{H}^{*}$ and $\chi_{L}^{H}(g)=0$.

Since $g_{H}^{*} \in \arg \max f_{H}(g)$, party $H$ has no incentive to deviate from proposing $\gamma^{H}(g)=$ $g_{H}^{*}$ and $\chi_{L}^{H}(g)=0$.

- $\underline{g}_{H} \leq g \leq \tilde{g}_{H}$ : In this case, $\gamma^{H}(g) \in\left[\theta_{H}+\theta_{L}, g_{H}^{*}\right]$ and $\chi_{L}^{H}(g)=0$.

We first show that proposing $\pi^{H}(g)$ is better than proposing $\left(\hat{g}, \hat{x}_{H}, \hat{x}_{L}\right)$ with $\hat{g}>\gamma^{H}(g)$ and then show that it is better than proposing $\left(\hat{g}, \hat{x}_{H}, \hat{x}_{L}\right)$ with $\hat{g}<\gamma^{H}(g)$.

$-\hat{g}>\gamma^{H}(g)$ : Since $\gamma^{H}(g)>\theta_{H}+\theta_{L}>\frac{\theta_{L}(1+\delta-2 \delta p)}{\delta(1-p)}$, by Claim B.5, for $\hat{g}>\gamma^{H}(g)$, $\alpha^{L}\left(g,\left(\hat{g}, \hat{x}_{H}, \hat{x}_{L}\right)\right)=1$ only if $\hat{x}_{L}>0$. Since party $L$ 's payoff is strictly decreasing in $x_{L}$, we only need to consider proposals such that the responder's acceptance constraint (5) is binding. Since $U_{H}^{P}(\hat{\mathbf{z}})$ is decreasing in $\hat{g}$ for $\hat{g}>\gamma^{H}(g) \geq \theta_{H}+\theta_{L}$ as shown before, the proposer has no incentive to make any proposal with $\hat{g}>\gamma^{H}(g)$.

$-\tilde{g}_{H} \leq \hat{g}<\gamma^{H}(g)$ : Consider $\hat{\mathbf{z}}=(\hat{g}, 1-\hat{g}, 0)$. Then $U_{H}^{P}(\hat{\mathbf{z}})=f_{H}(\hat{g})$. As shown in the proof of Claim B.4, $f_{H}(\hat{g})$ is increasing in $\hat{g}$ for $\tilde{g}_{H}<\hat{g}<g_{H}^{*}$. Since $\pi^{H}(g)=\left(\gamma^{H}(g), 1-\gamma^{H}(g), 0\right)$ where $\gamma^{H}(g)<g_{H}^{*}$, it follows that $U_{H}^{P}\left(\pi^{H}(g)\right)>$ $U_{H}^{P}(\hat{\mathbf{z}})$ for any $\hat{g}<\gamma^{H}(g) \leq g_{H}^{*}$. Since party $H^{\prime}$ 's payoff is decreasing in $x_{L}$, 
$U_{H}^{P}(\hat{\mathbf{z}}) \geq U_{H}^{P}\left(\left(\hat{g}, \hat{x}_{H}, \hat{x}_{L}\right)\right)$ for any $\left(\hat{g}, \hat{x}_{H}, \hat{x}_{L}\right) \in \mathcal{B}$, it follows that $U_{H}^{P}\left(\pi^{H}(g)\right)>$ $U_{H}^{P}\left(\left(\hat{g}, \hat{x}_{H}, \hat{x}_{L}\right)\right)$ for any $\hat{g}<\gamma^{H}(g) \leq g_{H}^{*}$. Hence the proposer has no incentive to deviate and make a proposal with $\tilde{g}_{H} \leq \hat{g}<\gamma^{H}(g)$.

$-g \leq \hat{g} \leq \tilde{g}_{H}$. Consider $\hat{\mathbf{z}}=(\hat{g}, 1-\hat{g}, 0)$. Then $U_{H}^{P}(\hat{\mathbf{z}})=f_{H}(\hat{g})$. Recall that for $g \geq g_{L}^{*}, f_{H}(g)=1-g+\frac{\theta_{H}(1+\delta-2 \delta p)}{1-\delta p} \ln (g)+\frac{\delta(p+\delta-2 \delta p)}{1-\delta p} V_{H}(g)$. Also, for $\underline{g}_{H} \leq \hat{g} \leq \tilde{g}, V_{H}(\hat{g})=V_{H}\left(\gamma^{H}(\hat{g})\right)$. Hence, $f_{H}\left(\gamma^{H}(\hat{g})\right)-f_{H}(\hat{g})=-\gamma^{H}(\hat{g})+$ $\hat{g}+\frac{\theta_{H}(1+\delta-2 \delta p)}{1-\delta p}\left(\ln \left(\gamma^{H}(\hat{g})\right)-\ln (\hat{g})>0\right.$ since $\hat{g} \leq \gamma^{H}(\hat{g}) \leq \frac{\theta_{H}(1+\delta-2 \delta p)}{1-\delta p}$. Since $\gamma^{H}(\hat{g})<\gamma^{H}(g)$ and $f_{H}(g)$ is increasing in $\left(\theta_{H}+\theta_{L}, g_{H}^{*}\right)$ as shown in the proof of Claim B.4, it follows that $f_{H}(\hat{g}) \leq f_{H}\left(\gamma^{H}(\hat{g})\right) \leq f_{H}\left(\gamma^{H}(g)\right)$ and therefore $U_{H}^{P}\left(\pi^{H}(g)\right) \geq U_{H}^{P}(\hat{\mathbf{z}})$ for any $\hat{g} \in\left[g, \tilde{g}_{H}\right]$. Hence proposing $\pi^{H}(g)$ is better than proposing any $\left(\hat{g}, \hat{x}_{H}, \hat{x}_{L}\right) \in \mathcal{B}$ with $g \leq \hat{g} \leq \tilde{g}_{H}$.

$-\hat{g}<g$ : By Corollary B. $2, g<\tilde{g}_{H}<\frac{\theta_{L}(1+\delta-2 \delta p)}{\delta(1-p)}$. Hence, for $\hat{g}<g, \alpha^{L}\left(g,\left(\hat{g}, \hat{x}_{H}, \hat{x}_{L}\right)\right)=$ 1 only if $\hat{x}_{L}>0$ by Claim B.5. Consider $\hat{\mathbf{z}}=\left(\hat{g}, \hat{x}_{H}, \hat{x}_{L}\right)$ such that (B26) holds. Since $U_{H}^{P}(\hat{\mathbf{z}})$ is increasing in $\hat{g}$ for $\hat{g}<g$ as shown before, the proposer has no incentive to deviate and make a proposal with $\hat{g}<g$.

- $\tilde{g}_{H} \leq g \leq \theta_{H}+\theta_{L}$ : In this case, $\gamma^{H}(g)=\theta_{H}+\theta_{L}$ and $\chi_{L}^{H}(g) \geq 0$.

Let $h(g)=\max \left\{g^{\prime} \in[0,1]: K_{L}\left(g^{\prime}\right)=K_{L}(g)\right\}$ and $l(g)=\min \left\{g^{\prime} \in[0,1]: K_{L}\left(g^{\prime}\right)=\right.$ $\left.K_{L}(g)\right\}$. By Claim B.5, $h(g) \in\left[\frac{1+\delta-2 \delta p}{\delta(1-p)} \theta_{L}, \theta_{H}+\theta_{L}\right]$ and $l(g) \in\left[\tilde{g}_{H}, \frac{1+\delta-2 \delta p}{\delta(1-p)} \theta_{L}\right]$.

$-\hat{g} \geq h(g)$ : For $\hat{\mathbf{z}}=\left(\hat{g}, \hat{x}_{H}, \hat{x}_{L}\right)$, Claim B.5 implies that $\alpha^{L}\left(g,\left(\hat{g}, \hat{x}_{H}, \hat{x}_{L}\right)\right)=1$ only if $\hat{x}_{L}>0$. Consider $\hat{\mathbf{z}}$ such that (B26) holds. As shown before, $U_{H}^{P}(\hat{g})$ is increasing for $\hat{g} \in\left[h(g), \theta_{H}+\theta_{L}\right)$ and decreasing for $\hat{g}>\theta_{H}+\theta_{L}$, and therefore the proposer has no incentive to deviate and make any proposal with $\hat{g} \geq h(g)$ and $\hat{g} \neq \theta_{H}+\theta_{L}$.

$-\hat{g} \in[l(g), h(g)]:$ Consider $\hat{\mathbf{z}}=(\hat{g}, 1-\hat{g}, 0)$. Since $U_{H}^{P}(\hat{\mathbf{z}})=f_{H}(\hat{g})$ and $f_{H}(\hat{\mathbf{z}})$ is increasing for $\hat{g} \in[l(g), h(g)]$, it follows that $U_{H}^{P}((h(g), 1-h(g), 0))>U_{H}^{P}(\hat{\mathbf{z}})$ for any $\hat{g} \in(l(g), h(g)$ and therefore the proposer has no incentive to deviate and make a proposal with $\hat{g} \in[l(g), h(g)]$.

$-\hat{g}<l(g)$ : For $\hat{\mathbf{z}}=\left(\hat{g}, \hat{x}_{H}, \hat{x}_{L}\right)$, Claim B.5 implies that $\alpha^{L}\left(g,\left(\hat{g}, \hat{x}_{H}, \hat{x}_{L}\right)\right)=1$ only if $\hat{x}_{L}>0$. Consider $\hat{\mathbf{z}}$ such that (B26) holds. As shown before, $U_{H}^{P}(\hat{g})$ is increasing for $\hat{g}<l(g)$, and therefore the proposer has no incentive to deviate and make any proposal with $\hat{g} \geq l(g)$.

- $g \in\left[\theta_{H}+\theta_{L}, g_{H}^{*}\right]:$ In this case, $\gamma^{H}(g)=g$ and $\chi_{L}^{H}(g)=0$. Recall that $l(g)=\min \left\{g^{\prime} \in\right.$ $\left.[0,1]: K_{L}\left(g^{\prime}\right)=K_{L}(g)\right\}$. In this case, $l(g) \in\left[\underline{g}_{H}, \tilde{g}_{H}\right]$.

$-\hat{g}>g$ : For $\hat{\mathbf{z}}=\left(\hat{g}, \hat{x}_{H}, \hat{x}_{L}\right)$, Claim B.5 implies that $\alpha^{L}\left(g,\left(\hat{g}, \hat{x}_{H}, \hat{x}_{L}\right)\right)=1$ only if $\hat{x}_{L}>0$. Consider $\hat{\mathbf{z}}$ such that (B26) holds. As shown before, $U_{H}^{P}(\hat{g})$ is decreasing for $\hat{g}>\theta_{H}+\theta_{L}$, and therefore the proposer has no incentive to deviate and make any proposal with $\hat{g} \geq g$. 
- $\tilde{g}_{H} \leq \hat{g}<g$ : Consider $\hat{\mathbf{z}}=(\hat{g}, 1-\hat{g}, 0)$. Since $U_{H}^{P}(\hat{\mathbf{z}})=f_{H}(\hat{g})$ and $f_{H}(\hat{g})$ is increasing if $\tilde{g}_{H} \leq \hat{g}<g$, it follows that the proposer has no incentive to deviate and make a proposal with $\hat{g} \in\left[\tilde{g}_{H}, g\right)$.

$-l(g) \leq \hat{g} \leq \tilde{g}_{H}$. Consider $\hat{\mathbf{z}}=(\hat{g}, 1-\hat{g}, 0)$. Note that for $\hat{g} \in\left[l(g), \tilde{g}_{H}\right], f_{H}(\hat{g})<$ $f_{H}\left(\gamma^{H}(\hat{g})\right)$. Also, since $\gamma^{H}(\hat{g})<g$ and therefore $f_{H}\left(\gamma^{H}(\hat{g})<f_{H}(g)\right.$, it follows that $f_{H}(\hat{g})<f_{H}(g)$. Hence the proposer has no incentive to deviate and make a proposal with $\hat{g} \in\left[l(g), \tilde{g}_{H}\right]$.

$-\hat{g} \leq l(g)$ : For $\hat{\mathbf{z}}=\left(\hat{g}, \hat{x}_{H}, \hat{x}_{L}\right)$, Claim B.5 implies that $\alpha^{L}\left(g,\left(\hat{g}, \hat{x}_{H}, \hat{x}_{L}\right)\right)=1$ only if $\hat{x}_{L}>0$. Consider $\hat{\mathbf{z}}$ such that (B26) holds. As shown before, $U_{H}^{P}(\hat{g})$ is increasing for $\hat{g} \leq l(g)$, and therefore the proposer has no incentive to deviate and make any proposal with $\hat{g} \leq l(g)$.

To summarize, party $H$ has no incentive to deviate from $\pi^{H}(g)$ for any $g \in[0,1]$.

B7. High-polarization equilibrium when condition (**) fails:

Figure B2 illustrates the parties' proposal strategies when condition $(* *)$ fails. Similar to the low-polarization case, $\left(\mathrm{G} 1^{\prime}\right)-\left(\mathrm{G} 4^{\prime}\right)$ are still satisfied in this figure except $\gamma^{L}(g)>\theta_{H}+\theta_{L}$ for very high status quos. As shown in Section V.B, the failure of condition (**) does not affect the set of steady states.

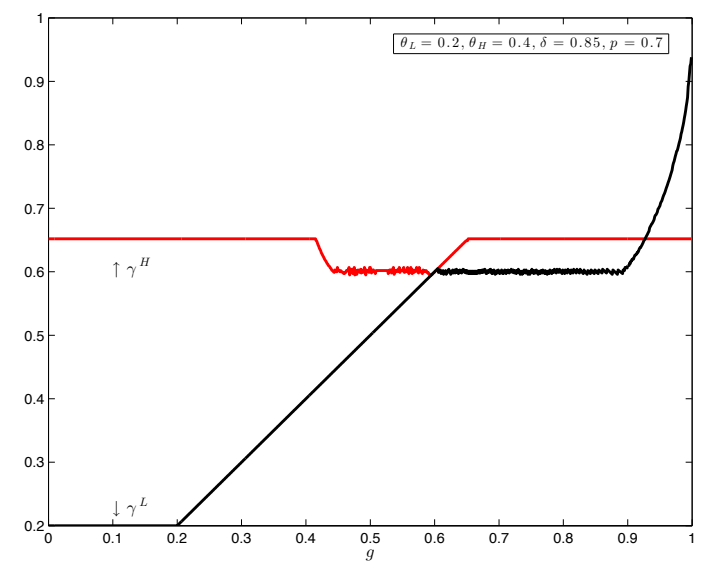

Figure B2. : $\gamma^{i}(g)$ in high-polarization case when condition $(* *)$ does not hold

B8. High-polarization equilibrium when $\psi<\theta_{L}^{*}$ :

Figure B3 illustrates the proposal strategies when $\psi<\theta_{L}^{*}$. In this case, polarization is very high. As the figure shows, two kinds of equilibria arise. In panel (a), the equilibrium strategies still satisfy $\left(\mathrm{G}^{\prime}\right)-\left(\mathrm{G} 4^{\prime}\right)$ with the exception that $g_{L}^{*}>\underline{g}_{H}$. In this case, party $L$ 's dynamic 
ideal is $g_{L}^{*}=\theta_{L}^{*}>\theta_{L}$, an analog to party $H$ 's dynamic ideal $g_{H}^{*}=\theta_{H}^{*}$. To understand the difference between Figure 5 and Figure B3(a), recall that $\underline{g}_{H}$ is the threshold below which party $L$ 's constraint is slack. In Figure $5, g_{L}^{*}=\theta_{L}<\underline{g}_{H}$, implying that party $L$ 's constraint is slack at its dynamic ideal, but in Figure B3(a), $\theta_{L}$ is greater than $\underline{g}_{H}$, implying that party $L$ 's choice of public good has a dynamic effect because if party $H$ comes to power in the next period, party $L$ 's constraint is binding. This dynamic effect results in party $L$ 's dynamic ideal $g_{L}^{*}$ being higher than its static ideal $\theta_{L}$. In panel (b), party $H$ 's strategy again satisfies the guesses, but party $L$ 's strategy violates $\left(\mathrm{G} 2^{\prime}\right)$. In particular, instead of proposing $g^{\prime}=g$, now party $L$ proposes a constant level $g^{\prime}=\theta_{L}^{*}$ when the status quo is in a subinterval of $\left[g_{L}^{*}, \theta_{H}+\theta_{L}\right]$ (see the kink in Figure B3(b)). By setting $g^{\prime}$ at a higher level $\theta_{L}^{*}$, party $L$ guarantees a higher bargaining position for itself in the next period. Hence if party $H$ comes to power in the next period, it is forced to set the efficient level of the public good rather than its dynamic ideal (which is much higher).

Although the details of party $L$ 's strategy violate certain aspects of $\left(\mathrm{G} 2^{\prime}\right)$ when $\psi<\theta_{L}^{*}$, the efficiency implications and the set of steady states are still the same, as illustrated in Figure B3 and formalized in Section V.B.

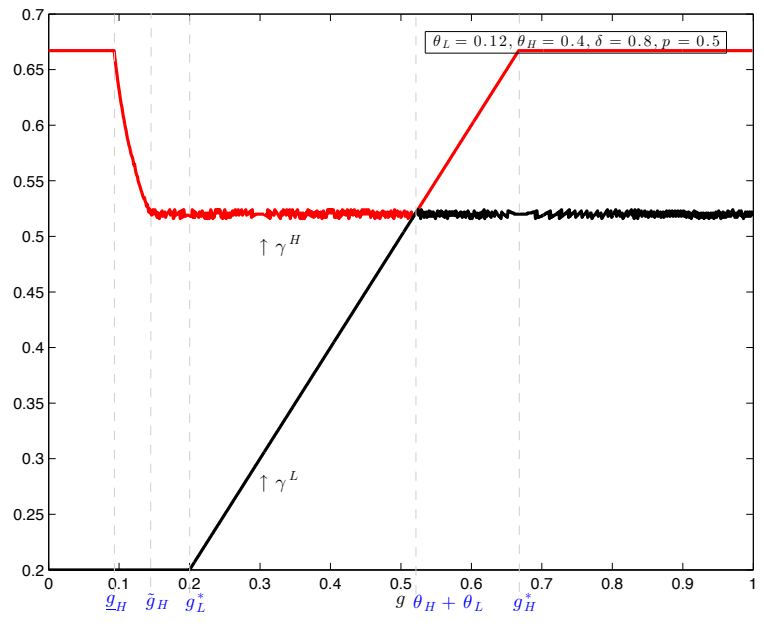

(a) $g_{L}^{*}=\theta_{L}^{*}>\theta_{L}$

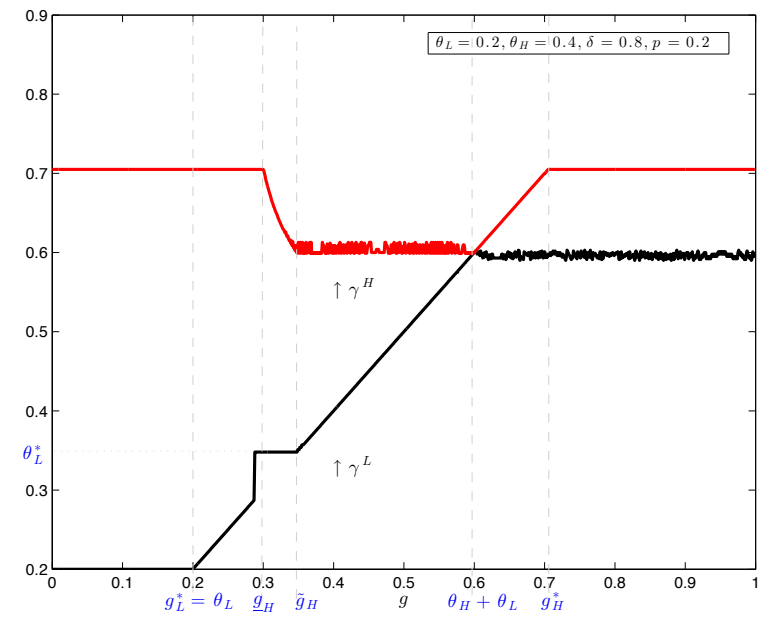

(b) $g_{L}^{*}=\theta_{L}$

Figure B3. : $\gamma^{i}(g)$ in high-polarization case when $\psi<\theta_{L}^{*}$

\section{B9. Proof of Proposition 8}

Let $\mathcal{S P E \mathcal { A }}$ denote the set of static Pareto efficient allocations.

CLAIM 3: An infinite repetition of an allocation $s \in \mathcal{S P E} \mathcal{A}$ is a dynamic Pareto efficient allocation. 
Proof: Suppose $\mathbf{s} \in \mathcal{S P \mathcal { E }} \mathcal{A}$. Then there exists $\alpha_{L} \geq 0$ and $\alpha_{H} \geq 0$ such that $\mathbf{s}$ is a solution to the following maximization problem:

$$
\max _{\mathbf{b} \in \mathcal{B}} \alpha_{L} u_{L}(\mathbf{b})+\alpha_{H} u_{H}(\mathbf{b}) .
$$

It follows that repeating $\mathbf{s}$ in every period is a solution to the following maximization problem:

$$
\max _{\left\{\mathbf{b}^{t}\right\}_{t=0}^{\infty} \in \mathcal{B}^{\infty}} \sum_{t=0}^{\infty} \delta^{t}\left(\alpha_{L} u_{L}\left(\mathbf{b}^{t}\right)+\alpha_{H} u_{H}\left(\mathbf{b}^{t}\right)\right),
$$

which is equivalent to

$$
\max _{\left\{\mathbf{b}^{t}\right\}_{t=0}^{\infty} \in \mathcal{B}^{\infty}} \alpha_{L} \sum_{t=0}^{\infty} \delta^{t} u_{L}\left(\mathbf{b}^{t}\right)+\alpha_{H} \sum_{t=0}^{\infty} \delta^{t} u_{H}\left(\mathbf{b}^{t}\right) .
$$

Hence, repeating $\mathbf{s}$ in every period is a dynamic Pareto efficient allocation.

CLAIM 4: Suppose $s \in \mathcal{S P \mathcal { A }}$. Then, (i) in any equilibrium in the infinite horizon game with all mandatory spending, $V_{i}(s)=\frac{1}{1-\delta} u_{i}(s)$ and $W_{i}(s)=\frac{1}{1-\delta} u_{i}(s)$ for $i \in\{H, L\}$, and (ii) there exists an equilibrium in the infinite horizon game with all mandatory spending in which $\pi^{i}(s)=s$ for $i \in\{H, L\}$.

Proof: First, note that for any status quo $\mathbf{s} \in \mathcal{B}$, proposing to maintain the status quo will be accepted by the responder. Hence, $V_{i}(\mathbf{s}) \geq \frac{1}{1-\delta} u_{i}(\mathbf{s})$ since when party $i$ is the proposer, it can maintain the status quo by proposing $\mathbf{s}$ and when the other party comes to power in the future, it can reject any proposal other than s. Similarly, $W_{i}(\mathbf{s}) \geq \frac{1}{1-\delta} u_{i}(\mathbf{s})$ since when party $i$ is the responder, it can maintain the status quo $\mathbf{s}$ by rejecting any proposal not equal to $\mathbf{s}$ and when it comes to power in the future, it can propose to maintain $\mathbf{s}$.

Since $\mathbf{s} \in \mathcal{S P \mathcal { E }} \mathcal{A}$, an infinite repetition of $\mathbf{s}$ is dynamically Pareto efficient by Claim 3 . Hence, $W_{j}(\mathbf{s}) \geq \frac{1}{1-\delta} u_{j}(\mathbf{s})$ implies that $V_{i}(\mathbf{s}) \leq \frac{1}{1-\delta} u_{i}(\mathbf{s})$ for $i \neq j$. Since $V_{i}(\mathbf{s}) \geq \frac{1}{1-\delta} u_{i}(\mathbf{s})$, we have $V_{i}(\mathbf{s})=\frac{1}{1-\delta} u_{i}(\mathbf{s})$ and $W_{j}(\mathbf{s})=\frac{1}{1-\delta} u_{j}(\mathbf{s})$ for $i \neq j$. It also follows that proposing to maintain $\mathbf{s}$ is a best response for party $i$. Hence, there exists an equilibrium in which $\pi^{i}(\mathbf{s})=\mathbf{s}$.

We immediately have the next result, which establishes the "if" part of Proposition 8.

CLAIM 5: If $s$ is a static Pareto efficient allocation, then there exists an equilibrium in which $s$ is a steady state.

We next show that only a static Pareto efficient allocation can be a steady state, establishing the "only if" part of Proposition 8.

CLAIM 6: If $s$ is not a static Pareto efficient allocation, then there is no equilibrium in which $s$ is a steady state. 
Proof: Suppose to the contrary that there exists an equilibrium and a state $\mathbf{s} \notin \mathcal{S P \mathcal { A }} \mathcal{A}$ such that $\pi^{i}(\mathbf{s})=\mathbf{s}$ in this equilibrium. Then, $V_{i}(\mathbf{s})=W_{i}(\mathbf{s})=K_{i}(\mathbf{s})=\frac{1}{1-\delta} u_{i}(\mathbf{s})$ in this equilibrium

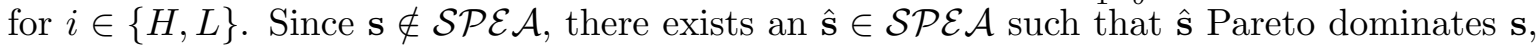
that is, $u_{i}(\hat{\mathbf{s}})>u_{i}(\mathbf{s}), u_{j}(\hat{\mathbf{s}}) \geq u_{j}(\mathbf{s})$ for some $i \in\{H, L\}$ and $j \neq i$. Suppose party $i$ proposes $\hat{\mathbf{s}}$ when the status quo is $\mathbf{s}$. If the responder $j$ accepts, its payoff is $u_{j}(\hat{\mathbf{s}})+\delta\left[(1-p) V_{j}(\hat{\mathbf{s}})+p W_{j}(\hat{\mathbf{s}})\right]$. Since $\hat{\mathbf{s}} \in \mathcal{S P \mathcal { E }} \mathcal{A}, V_{j}(\hat{\mathbf{s}})=W_{j}(\hat{\mathbf{s}})=\frac{1}{1-\delta} u_{j}(\hat{\mathbf{s}})$ in any equilibrium by Claim 4 . Hence,

$$
u_{j}(\hat{\mathbf{s}})+\delta\left[(1-p) V_{j}(\hat{\mathbf{s}})+p W_{j}(\hat{\mathbf{s}})\right]=\frac{1}{1-\delta} u_{j}(\hat{\mathbf{s}}) \geq K_{j}(\mathbf{s}),
$$

and therefore responder $j$ accepts $\hat{\mathbf{s}}$ when the status quo is $\mathbf{s}$. It follows that party $i$ 's payoff by proposing $\hat{\mathbf{s}}$ when the status quo is $\mathbf{s}$ is

$$
u_{i}(\hat{\mathbf{s}})+\delta\left[p V_{i}(\hat{\mathbf{s}})+p W_{i}(\hat{\mathbf{s}})\right]=\frac{1}{1-\delta} u_{i}(\hat{\mathbf{s}})>V_{i}(\mathbf{s}),
$$

a contradiction. Hence, there is no equilibrium in which $\mathbf{s} \notin \mathcal{S P \mathcal { E }} \mathcal{A}$ is a steady state. 
B10. Illustration of all mandatory equilibrium proposals

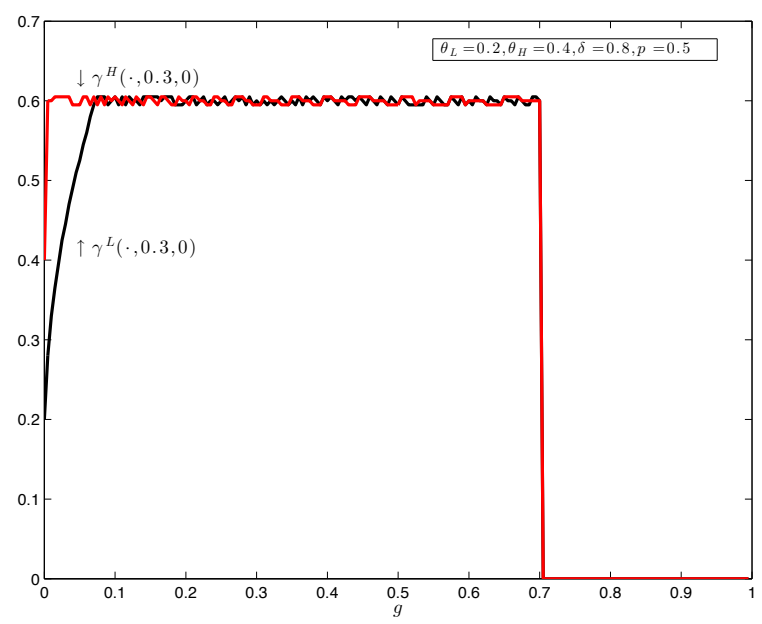

Figure B4. : $\gamma^{i}(g, 0.3,0)$

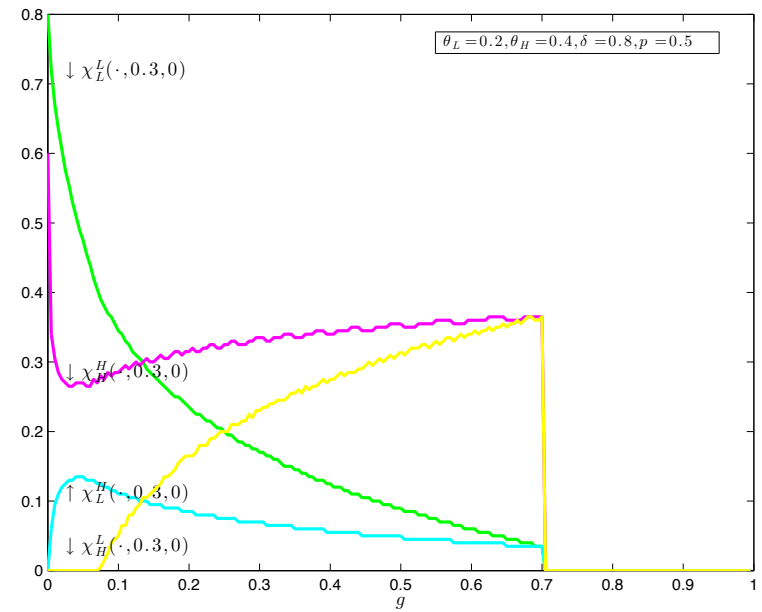

Figure B5. : $\chi_{j}^{i}(g, 0.3,0)$ 


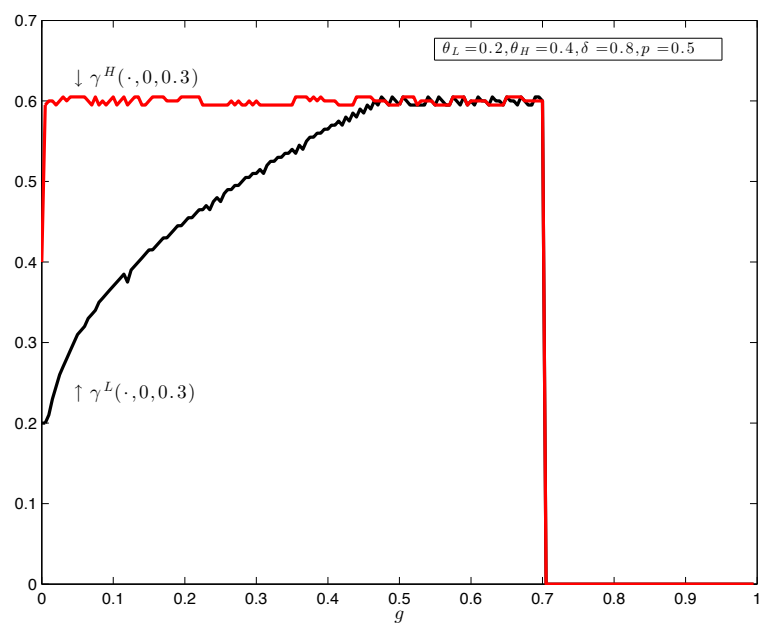

Figure B6. : $\gamma^{i}(g, 0,0.3)$

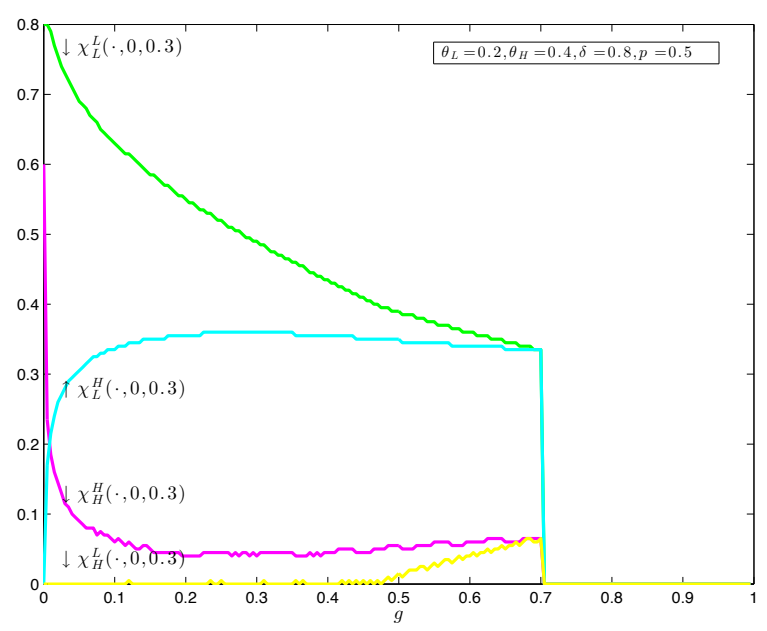

Figure B7. : $\chi_{j}^{i}(g, 0,0.3)$ 